\title{
AUTOMORPHISM GROUPS OF COUNTABLE ARITHMETICALLY SATURATED MODELS OF PEANO ARITHMETIC
}

\author{
JAMES H. SCHMERL
}

\begin{abstract}
If $\mathcal{M}, \mathcal{N}$ are countable, arithmetically saturated models of Peano Arithmetic and $\operatorname{Aut}(\mathcal{M}) \cong \operatorname{Aut}(\mathcal{N})$, then the Turingjumps of $\operatorname{Th}(\mathcal{M})$ and $\operatorname{Th}(\mathcal{N})$ are recursively equivalent.
\end{abstract}

Since 1991, when the question

Are there countable, recursively saturated models $\mathcal{M}$, $\mathcal{N}$ of PA such that $\operatorname{Aut}(\mathcal{M}) \neq \operatorname{Aut}(\mathcal{N})$ (as abstract groups)?

appeared in [8], it has been of interest to determine to what extent (the isomorphism type of) the $\operatorname{group} \operatorname{Aut}(\mathcal{M})$ of all automorphisms of a countable, recursively saturated model $\mathcal{M}$ of Peano Arithmetic determines (the isomorphism type of) $\mathcal{M}$. It was proved in 8 that whenever both $\mathcal{M}$ and $\mathcal{N}$ are countable, recursively saturated models of PA and exactly one of them is arithmetically saturated, then $\operatorname{Aut}(\mathcal{M})$ and $\operatorname{Aut}(\mathcal{N})$ are not isomorphic as topological groups. In 1994, Lascar [16] proved that countable, arithmetically saturated models of PA have the small index property, and that result then implied that $\operatorname{Aut}(\mathcal{M}) \mp \operatorname{Aut}(\mathcal{N})$ as abstract groups. This gave the first positive answer to the above question. A neater way, in which the use of the small index property is masked, that automorphism groups distinguish those models that are arithmetically saturated from the other countable recursively saturated ones was obtained the next year in [13, Coro. 3.9] (or see [15, Th. 9.3.10]): If $\mathcal{M}$ is countable and recursively saturated, then $\mathcal{M}$ is arithmetically saturated iff the cofinality of $\operatorname{Aut}(\mathcal{M})$ is uncountable. Finally, we mention that Kaye's Theorem [7] (see §1) characterizing the closed normal subgroups of $\operatorname{Aut}(\mathcal{M})$, which appeared in the same volume [9] as did Lascar's Theorem, yields that whenever $\mathcal{M}, \mathcal{N}$ are countable, arithmetically saturated models and $\mathcal{M}$ is a model of True Arithmetic (TA) while $\mathcal{N}$ is not, then $\operatorname{Aut}(\mathcal{M}) \nsucceq \operatorname{Aut}(\mathcal{N})$.

Date: June 10, 2018. 
Recall that a countable, recursively saturated model $\mathcal{M}$ of PA is determined up to isomorphism by two invariants: its standard system $\operatorname{SSy}(\mathcal{M})$ and its first-order theory $\operatorname{Th}(\mathcal{M})$. Correspondingly, there are the following complementary questions for a countable, recursively saturated model $\mathcal{M}$ of PA.

To what extent does $\operatorname{Aut}(\mathcal{M})$ determine $\operatorname{SSy}(\mathcal{M})$ ? $\operatorname{Th}(\mathcal{M})$ ?

Subsequent to Lascar's proof about the small index property, the focus has been almost entirely on countable, arithmetically saturated models. The "SSy" question for these models was answered soon thereafter.

TheOREM 1: (Kossak-Schmerl [14]) If $\mathcal{M}, \mathcal{N}$ are countable, arithmetically saturated models of PA such that $\operatorname{Aut}(\mathcal{M}) \cong \operatorname{Aut}(\mathcal{N})$, then $\operatorname{SSy}(\mathcal{M})=\operatorname{SSy}(\mathcal{N})$.

Progress on the "Th" question has been slower. Nurkhaidarov [18] proved the following in 2006.

ThEOREM 2: (Nurkhaidarov [18]) There are countable, arithmetically saturated models $\mathcal{M}_{0}, \mathcal{M}_{1}, \mathcal{M}_{2}, \mathcal{M}_{3}$ of PA such that whenever $i<j<4$, then $\operatorname{SSy}\left(\mathcal{M}_{i}\right)=\operatorname{SSy}\left(\mathcal{M}_{j}\right)$ and $\operatorname{Aut}\left(\mathcal{M}_{i}\right) \not \operatorname{Aut}\left(\mathcal{M}_{j}\right)$.

Although not explicitly stated in [18], the proof of Theorem 2 also proves the following stronger result.

TheOREM 3: (Nurkhaidarov [18]) There are completions $T_{0}, T_{1}, T_{2}$, $T_{3}$ of PA such that whenever $i<j<4$ and $\mathcal{M}_{i}, \mathcal{M}_{j}$ are countable, arithmetically saturated models of $T_{i}, T_{j}$, respectively, then $\operatorname{Aut}\left(\mathcal{M}_{i}\right) ¥$ $\operatorname{Aut}\left(\mathcal{M}_{j}\right)$.

Theorem 3 implies Theorem 2 because whenever $\mathcal{T}$ is a countable set of completions of PA, then there is an $\mathfrak{X}$ such that each $T \in \mathcal{T}$ has a countable, arithmetically saturated model whose standard system is $\mathfrak{X}$.

This paper improves Theorem 3 by increasing the cardinal number 4 in that theorem to the maximum possible of $2^{\aleph_{0}}$. If $X, Y \subseteq \omega$, then we write $X \leq_{T} Y$ if $X$ is Turing-reducible (or recursive relative) to $Y$, and $X \equiv_{T} Y$ if $X$ is recursively equivalent to $T$ (that is, $X \leq_{T} Y \leq_{T} X$ ). As usual, the Turing-jump of $X$ is $X^{\prime}$. The following theorem is our principal new result.

THEOREM 4: If $\mathcal{M}, \mathcal{N}$ are countable, arithmetically saturated models of $\operatorname{PA}$ and $\operatorname{Aut}(\mathcal{M}) \cong \operatorname{Aut}(\mathcal{N})$, then $\operatorname{Th}(\mathcal{M})^{\prime} \equiv_{T} \operatorname{Th}(\mathcal{N})^{\prime}$.

A consequence of this theorem is that the cardinal 4 in Theorem 2 can be increased to the maximum possible of $\aleph_{0}$. In fact, we get the 
following corollary that yields some answers to Question 15 in [15, Chap. 12].

COROLlary 5: For any countable jump ideal $\mathfrak{X}$, there are infinitely many countable arithmetically saturated models $\mathcal{M}_{0}, \mathcal{M}_{1}, \mathcal{M}_{2}, \ldots$ of PA such that whenever $i<j<\omega$, then $\operatorname{Aut}\left(\mathcal{M}_{i}\right) ¥ \operatorname{Aut}\left(\mathcal{M}_{j}\right)$ and $\operatorname{SSy}\left(\mathcal{M}_{i}\right)=\mathfrak{X}$.

One may wonder whether Theorems 1 and 4 tell the whole story. In other words, if $\mathcal{M}, \mathcal{N}$ are countable, arithmetically saturated models of PA such that $\operatorname{Aut}(\mathcal{M}) \cong \operatorname{Aut}(\mathcal{N})$, then is it the case that $\operatorname{SSy}(\mathcal{M})=$ $\operatorname{SSy}(\mathcal{N})$ and $\operatorname{Th}(\mathcal{M})^{\prime} \equiv_{T} \operatorname{Th}(\mathcal{N})^{\prime}$ ? We easily see that this is not so since the 4 theories in Theorem 3 can be chosen to be recursively equivalent. However, we can do even better.

THEOREM 6: For each $n<\omega$, there are recursively equivalent completions $T_{0}, T_{1}, \ldots, T_{n}$ of PA such that whenever $i<j \leq n$ and $\mathcal{M}_{i}, \mathcal{M}_{j}$ are countable, arithmetically saturated models of $T_{i}, T_{j}$, respectively, then $\operatorname{Aut}\left(\mathcal{M}_{i}\right) \approx \operatorname{Aut}\left(\mathcal{M}_{j}\right)$.

The results presented in this paper suggest the question that is dual to the one asked in [8] and could have just as easily been asked there.

Question 7: Are there countable, recursively saturated models $\mathcal{M}$, $\mathcal{N}$ of PA such that $\mathcal{M} \approx \mathcal{N}$ and $\operatorname{Aut}(\mathcal{M}) \cong \operatorname{Aut}(\mathcal{N})$ ?

The analogous question for countable, arithmetically saturated models is also open.

There are 6 sections that follow this introductory one. Some preliminaries are in $\S 1$, which consists of some notation, definitions and results that will be used in the succeeding sections. The next two sections do not overtly refer to automorphism groups. Some special types of models of PA, the lofty models and those with the $\omega$-property, are discussed in $\S 2$. In $\S 3$, we consider substructure lattices, carefully reviewing a result from [13]. Our main result, Theorem 4, is proved in $\S 4$. Theorem 6 is proved in $\S 5$, and some additional results are given in $\S 6$.

Roman Kossak and Ermek Nurkhaidarov are thanked for their helpful comments on various precursors of this paper.

§1. Some Preliminaries. Notation and terminology used here will generally follow [15]. The reader should refer to [15] for insufficiently explained notions. The proof of Theorem 4 relies on a number of results 
that are proved or stated in [19]. It is suggested that the reader have that paper available.

The language appropriate for $\mathrm{PA}$ is $\mathcal{L}_{\mathrm{PA}}=\{+, \times, \leq 0,1\}$. It is to be tacitly understood that all models referred to in this paper are models of PA. All models are assumed to have the standard model $\mathbb{N}=$ $(\omega,+, \times, \leq, 0,1)$ as a submodel. Models will be denoted by (possibly adorned) script letters such as $\mathcal{M}, \mathcal{N}, \mathcal{M}_{1}, \ldots$, and their universes are denoted by the corresponding roman letters $M, N, M_{1}, \ldots$, although models and their universes may occasionally be confounded.

Suppose that $X, Y \subseteq \omega$. As already mentioned, $X \leq_{T} Y$ iff $X$ is Turing-reducible to $Y, X \equiv Y$ iff $X$ and $Y$ are recursively equivalent, and $X^{\prime}$ is the Turing-jump of $X$. For a small ordinal $\alpha, X^{(\alpha)}$ is the $\alpha$-th jump of $X$. If there is $n<\omega$ such that $X \leq_{T} Y^{(n)}$, then $X$ is arithmetically reducible to $Y$ (in symbols: $X \leq_{a} Y$ ). If $X \leq_{a} Y \leq_{a} X$, then $X$ and $Y$ are arithmetically equivalent (in symbols: $X \equiv_{a} Y$ ). We let $X \oplus Y=\{2 x: x \in X\} \cup\{2 y+1: y \in Y\}$.

A Turing ideal is a nonempty subset $\mathfrak{X} \subseteq \mathcal{P}(\omega)$ such that whenever $X, Y \in \mathfrak{X}$ and $Z \leq_{T} X \oplus Y$, then $Z \in \mathfrak{X}$. A jump ideal is a Turing ideal $\mathfrak{X}$ such that $Y \in \mathfrak{X}$ whenever $Y \leq_{a} X \in \mathfrak{X}$.

Suppose that $\mathcal{M}$ is an arbitrary model. If $A \subseteq M$, then the model generated by $A$, denoted by $\operatorname{Scl}(A)$, is the smallest elementary substructure of $\mathcal{M}$ containing $A$. The model $\mathcal{M}$ is finitely generated iff $\mathcal{M}=\operatorname{Scl}(a)(=\operatorname{Scl}(\{a\}))$ for some $a \in M$. A subset $I$ is a cut of $\mathcal{M}$ iff $0 \in I$ and whenever $x \leq y \in I$, then $x+1 \in I$. A cut $I$ is invariant iff $I=\sup (I \cap \operatorname{Scl}(0))$ or $I=\inf (\operatorname{Scl}(0) \backslash I)$, and it is exponentially closed iff $2^{x} \in I$ whenever $x \in I$. We let $\operatorname{Lt}(\mathcal{M})$ be the lattice of elementary substructures of $\mathcal{M}$ and $\operatorname{Lt}_{0}(\mathcal{M})$ be its $\vee$-subsemilattice consisting of those models in $\operatorname{Lt}(\mathcal{M})$ that are finitely generated. It is a consequence of Ehrenfeucht's Lemma ([15, Theorem 1.7.2]) that $\operatorname{Aut}(\mathcal{M})$ is trivial whenever $\mathcal{M}$ is finitely generated. The standard system of $\mathcal{M}$ is $\operatorname{SSy}(\mathcal{M})=\{D \cap \omega: D$ is a definable subset of $\mathcal{M}\}$. In general, $\operatorname{SSy}(\mathcal{M})$ is a $\operatorname{Scott}$ set or, equivalently, $(\mathbb{N}, \operatorname{SSy}(\mathcal{M})) \models \mathrm{WKL}_{0}$. If $\mathcal{M}$ is recursively saturated, then $\mathcal{M}$ is arithmetically saturated iff $(\mathbb{N}, \operatorname{SSy}(\mathcal{M})) \models \operatorname{ACA}_{0}$ iff $(\mathbb{N}, \operatorname{SSy}(\mathcal{M})) \models \mathrm{RT}_{2}^{3}$ iff $\operatorname{SSy}(\mathcal{M})$ is a jump ideal iff $\omega$ is a strong cut of $\mathcal{M}$. Here, we are letting $\mathrm{RT}_{2}^{n}$ denote infinite Ramsey's Theorem for 2-colored $n$-sets.

The usual interval notation will be used. If $\mathcal{M}$ is a model and $a, b \in$ $M$, then $[a, b]=\{x \in M: a \leq x \leq b\}$ and $[a, b)=\{x \in M: a \leq x<$ b\}.

If $T \supseteq$ PA is a theory (which, for us, is a (not necessarily deductively closed) consistent set of sentences) and $X \subseteq \omega$, then $X$ is a real represented by $T$ if there is a unary formula $\varphi(x)$ such that for each 
$n<\omega$,

$$
n \in X \Longleftrightarrow T \vdash \varphi(n) \Longleftrightarrow T \forall \neg \varphi(n) .
$$

As usual, $\operatorname{Rep}(T)$ is the set of reals represented by $T$. If $T$ is complete, then $\operatorname{Rep}(T)$ is the standard system of the prime model of $T$.

If $G=\operatorname{Aut}(\mathcal{M})$ and $A \subseteq M$, then the pointwise stabilizer of $A$ is the subgroup $G_{(A)}=\{g \in G: g(a)=a$ for all $a \in A\}$ and the setwise stabilizer is $G_{\{A\}}=\{g \in G: g[A]=A\}$. If $A=\{a\}$, then $G_{a}=G_{(A)}=$ $G_{\{A\}}$. When considering $G$ as a topological group, the stabilizers of finite subsets of $M$ are its basic open subgroups. Equivalently, the basic open subgroups are the pointwise stabilizers of finitely generated elementary submodels. Since finitely generated models do not have any nontrivial automorphisms, the basic open subgroups are also the setwise stabilizers of finitely generated elementary submodels.

The following theorem has already been mentioned.

KAYE'S THEOREM: If $\mathcal{M}$ is a countable recursively saturated model and $H \leq G=\operatorname{Aut}(\mathcal{M})$, then the following are equivalent:

(1) $H$ is a closed normal subgroup of $G$.

(2) $H=G_{(I)}$, where $I \subseteq M$ is an invariant, exponentially closed cut.

Even though the next theorem will be not explicitly used in this paper, we state it since it shows that for the implication $(1) \Longrightarrow(2)$ in Kaye's Theorem there is a unique such $I$.

SMORYŃSKI'S ThEOREM: Suppose that $\mathcal{M}$ is a countable recursively saturated model and that $H \leq G=\operatorname{Aut}(\mathcal{M})$. If $H=G_{(I)}$ for some cut $I \subseteq M$, then there is a unique exponentially closed cut $J \subseteq M$ such that $H=G_{(J)}$.

Smoryński actually proved more (see [15, Theorem 8.4.2]): If $\mathcal{M}$ is a countable recursively saturated model and $I \subseteq M$ is an exponentially closed cut, then there is $f \in \operatorname{Aut}(\mathcal{M})$ such that $I=I_{f i x}(f)=\{x \in M$ : $f(y)=y$ for all $y<x\}$.

For the record, we state Lascar's Theorem on the small index property.

LASCAR'S THEOREM: Suppose that $\mathcal{M}$ is a countable, arithmetically saturated model. If $H \leq \operatorname{Aut}(\mathcal{M})$, then $H$ is open iff its index $|\operatorname{Aut}(\mathcal{M}): H|$ is countable.

Scott [22] introduced the notion of a Scott set and proved two related theorems concerning standard systems and sets of represented reals. 
We will need the following variants of these two theorems, the first of which also appears as [6, Theorem 13.6].

TheOREM 1.1: (Wilmers [24]) Suppose that $T$ is a completion of PA and $\mathfrak{X}$ is a set of subsets of $\omega$. The following are equivalent:

(1) $\mathfrak{X}$ is a countable Scott set and $T \in \mathfrak{X}$.

(2) There is a countable recursively saturated $\mathcal{M} \models T$ such that $\operatorname{SSy}(\mathcal{M})=\mathfrak{X}$.

If $X \subseteq \omega$ and $i<\omega$, then $(X)_{i}=\{j<\omega:\langle i, j\rangle \in X\}$. If $\mathfrak{X}$ is a set of subsets of $\omega$ and $X \subseteq \omega$, then $\mathfrak{X}$ is enumerated by $X$ if $\mathfrak{X}=\left\{(X)_{i}: i<\omega\right\}$.

Theorem 1.2: (Knight [10, Coro. 1.6] and D. Marker) If $\mathfrak{X}$ is a Scott set enumerated by $X$ and $X \leq_{T} Y$, then PA has a completion $T$ such that $\operatorname{Rep}(T)=\mathfrak{X}$ and $T \equiv_{T} Y$.

The next proposition collects together some well known properties of countable, recursively saturated models most of which can be found in various places in [15].

Proposition 1.3: Suppose that $\mathcal{M}$ is a countable, recursively saturated model.

(1) $\mathcal{M}$ is tall.

(2) $\mathcal{M}$ is generated by a set of indiscernibles of any countable order type with no last element.

(3) $\mathcal{M}$ is homogeneous.

(4) If $\mathcal{M}_{0} \succ_{\mathrm{cf}} \mathcal{M}$, then $\mathcal{M}_{0}$ is recursively saturated.

(5) There is $\mathcal{M}_{0} \prec_{\text {end }} \mathcal{M}$ such that $\mathcal{M}_{0} \cong \mathcal{M}$.

What follows in this paragraph is a small exception to our convention that all models considered here are models of PA. Consider the language $\mathcal{L}^{*}=\mathcal{L}_{\mathrm{PA}} \cup\{U\}$, where $U$ is a new unary relation symbol, and then let $\mathrm{PA}^{*}$ be the $\mathcal{L}^{*}$-theory obtained from PA by adjoining all instances of the induction scheme in this expanded language. Each model of PA* expands a model of PA. Every statement in this paper that applies to models of PA has a natural extension that applies to models of PA*. We will have several occasions when we will want to refer to such an extended version of some result, and we will do so by referring to its *-version.

$\S 2$. Loftiness and the $\omega$-property. This section is concerned with some properties of models that were introduced in [4], [5] and 
[11]. Results of this section will be used in the proofs of the main results although automorphism groups do not appear here. The results may be of independent interest. Theorem 2.8 has to do with constructing countable models that have the $\omega$-property but are not recursively saturated. Corollary 2.9 is a common generalization of Proposition 1.3(2),(4) and (5). We begin with the definitions.

Definition 2.1: Suppose that $\mathcal{M}$ is a nonstandard model and $I$ is a cut.

(1) $I$ is upward monotonically $\omega$-lofty if there is $a \in M$ such that $I=\sup \left\{(a)_{i}: i<\omega\right\}$.

(2) $I$ is downward monotonically $\omega$-lofty if there is $a \in M$ such that $I=\inf \left\{(a)_{i}: i<\omega\right\}$.

(3) $I$ is uniformly $\omega$-lofty if there is $a \in M$ such that whenever $p<I<q$, then $p<(a)_{i}<q$ for some $i<\omega$.

(4) $\mathcal{M}$ is uniformly $\omega$-lofty if for any $b \in M$ there is $a \in M$ such that whenever $\omega<e \in M$, then $\operatorname{Scl}(b) \subseteq\left\{(a)_{i}: i<e\right\}$.

(5) $\mathcal{M}$ has the $\omega$-property if there is $\mathcal{N} \succ$ end $\mathcal{M}$ such that $M$ is an upward monotonically $\omega$-lofty cut of $\mathcal{N}$.

Various notions of loftiness were introduced and studied in [4] and [5]. Definitions (1), (2) and (3) are from [5, Def. 3.1]. Definition (4) is not the one given in [4, Def. 1.4(iii)], although it is equivalent. One direction of this equivalence is given in [13, Theorem 1.7(1b)]; the other is easy to see. It is straightforward to see that every recursively saturated model is uniformly $\omega$-lofty. In fact, if we let $t_{0}(x), t_{1}(x), t_{2}(x), \ldots$ be a recursive list of all Skolem terms, then $\mathcal{M}$ is recursively saturated iff for every $b \in M$ there is $a \in M$ such that $\mathcal{M} \models(a)_{i}=t_{i}(b)$ for all $i<\omega$ ([5, Prop. 1.6]). Clearly, every uniformly $\omega$-lofty model is tall. Finally, (5) was introduced by Kossak in [11] and studied by him in [11] and [12].

According to [11], the following lemma is implicit in [5]; it is explicitly proved in [11, Theorem 2.7].

Lemma 2.2: If $\mathcal{M}$ is tall and has the $\omega$-property, then $\mathcal{M}$ is uniformly $\omega$-lofty.

Lemma 2.3: Suppose that $\mathcal{M}$ is countable and uniformly $\omega$-lofty. The following are equivalent:

(1) $\mathcal{M}$ is recursively saturated.

(2) $\mathcal{M}$ is generated by a set of indiscernibles.

(3) $\mathcal{M}$ is isomorphic to some $\mathcal{M}_{0} \prec_{\text {end }} \mathcal{M}$. 
Proof. (1) $\Longrightarrow(2)$ and (1) $\Longrightarrow(3)$ are (2) and (5) of Proposition 1.3.

For the converses, we rely on [5, Lemma 1.8 - Theorem 1.13] from which it follows that if $\mathcal{M}_{0} \prec_{\text {end }} \mathcal{M}$ and $\mathcal{M}_{0}$ is not recursively saturated, then there is $a \in M \backslash M_{0}$ that realizes a type not realized in $\mathcal{M}_{0}$. Both of the implications $(2) \Longrightarrow(1)$ and $(3) \Longrightarrow(1)$ are easy consequences.

Suppose that $\mathcal{M} \prec \mathcal{N}$ and $I$ is a cut of $\mathcal{M}$. We say that $\mathcal{N}$ fills the cut $I$ if there is $b \in N$ such that $I=\{a \in M: \mathcal{N} \models a<b\}$. In case $I=\omega$, we use the notation $\mathcal{M} \prec^{\omega} \mathcal{N}$ to indicate that $\mathcal{N}$ fills $\omega$. For us, the significance of this definition is the following well known equivalence: If $\mathcal{M}$ is a countable, nonstandard model, then $\operatorname{SSy}(\mathcal{M})$ is a jump ideal iff there is $\mathcal{N}$ such that $\mathcal{M} \prec^{\omega} \mathcal{N}$ and $\operatorname{SSy}(\mathcal{M})=\operatorname{SSy}(\mathcal{N})$. See, for example, [15, Theorem 7.3.4].

The next lemma is Lemma 3.11 of [5].

LEMma 2.4: Suppose that $\mathcal{M}$ is a countable model and $I$ is a proper cut of $\mathcal{M}$. If $I$ is not downward monotonically $\omega$-lofty, then there is a countable $\mathcal{N} \succ_{\text {cf }} \mathcal{M}$ such that $\mathcal{N} \nsucc^{\omega} \mathcal{M}$ and $\sup ^{\mathcal{N}}(I)$ is an upward monotonically $\omega$-lofty cut of $\mathcal{N}$.

Proof. We sketch the proof as it will be needed later on. The key notion that is used in the proof of Lemma 3.11 of [5] is that of an $\langle S, Q\rangle$-rich set ([5, Def. 3.6]). We say that $C$ is $\langle S, Q\rangle$-rich if $C, S, Q \in$ $\operatorname{Def}(\mathcal{M}), S$ is bounded, and whenever $f: S \longrightarrow Q$ is definable in $\mathcal{M}$, then there is $c \in C$ such that $(c)_{i}=f(i)$ for every $i \in S$.

Suppose that $I$ is not downward monotonically $\omega$-lofty.

The key combinatorial fact that is used in the proof of Lemma 3.11 is the following:

FACT 1: ([5, Lemma 3.10]) Suppose that $r<\omega<s \in M, I<q \in M$, $C$ is $\langle[r, s),[0, q)\rangle$-rich, and $g: M \longrightarrow M$ is definable. Then one of the following holds:

(1) There are $n, t<\omega$ and $\left\{d_{i}: i \in[r, t)\right\} \subseteq I$ such that $r \leq t$ and $\left\{c \in C: g(c) \leq n\right.$ and $(c)_{i}=d_{i}$ for $\left.r \leq i<t\right\}$ is $\langle[t, s),[0, q)\rangle-$ rich.

(2) There are $n, v, u \in M$ such that $n>\omega, \omega<v \leq s, I<u \leq q$ and $\{c \in C: g(c)>n\}$ is $\langle[r, v),[0, u)\rangle$-rich.

The proof then proceeds as follows. Choose arbitrary $s_{0}=q_{0}>I$, and let $r_{0}=0$ and $C_{0}=M$. Obtain a decreasing sequence $C_{0} \supseteq C_{1} \supseteq$ $C_{2} \supseteq \cdots$ such that each $C_{i}$ is $\left\langle\left[r_{i}, s_{i}\right),\left[0, q_{i}\right)\right\rangle$-rich, where $r_{i}<\omega<s_{i}$ and $I<q_{i}$. Also, there are $d_{0}, d_{1}, \ldots, d_{r_{i}-1} \in I$ such that whenever 
$c \in C_{i}$ and $j<r_{i}$, then $(c)_{j}=d_{j}$. Furthermore, for each definable $g: M \longrightarrow M$ there is an $i<\omega$ such that $C_{i+1}$ is obtained from $C_{i}$ in the obvious way using Fact 1 . And finally, for each $d \in I$ there are $i, n<\omega$ such that $(c)_{n}=d$ for all $c \in C_{i}$. This sequence determines a type over $\mathcal{M}$. Then, let $\mathcal{N}$ be an elementary extension of $\mathcal{M}$ generated by an element $c$ realizing this type. Clearly, $I=\left\{(c)_{n}: n<\omega\right\}$, so $\sup ^{\mathcal{N}}(I)$ is upward monotonically $\omega$-lofty.

We get the following corollary (to be improved by Corollary 2.7 and again by Theorem 2.8).

Corollary 2.5: Suppose that $\mathcal{M}$ is nonstandard and countable. Then there is $\mathcal{N} \succ_{\text {cf }} \mathcal{M}$ such that $\mathcal{N} \nsucc^{\omega} \mathcal{M}$ and $\mathcal{N}$ has the $\omega$-property.

Proof. Let $\mathcal{M}_{0} \succ_{\text {end }} \mathcal{M}$ be such that $\mathcal{M}_{0}$ is countable and $M$ is not a downward monotonically $\omega$-lofty cut of $\mathcal{M}_{0}$. (For example, let $\mathcal{M}_{0}$ be a countable, conservative extension of $\mathcal{M}$.) Apply Lemma 2.4 to get a countable $\mathcal{N}_{0} \succ_{\text {cf }} \mathcal{M}_{0}$ such that $\sup ^{\mathcal{N}_{0}}(M)$ is an upward monotonically $\omega$-lofty cut of $\mathcal{N}_{0}$. Then the unique $\mathcal{N}$ such that $\mathcal{M} \preccurlyeq$ cf $\mathcal{N} \prec_{\text {end }} \mathcal{N}_{0}$ is as required.

A construction, due to Paris, of a countable $\mathcal{N}$ that has the $\omega$ property but is not recursively saturated is presented in 11, Theorem 3.2]. Another way of getting such a model $\mathcal{N}$ is by use of the previous corollary. First, we need a definition taken from [4, page 111] (or see [5, Notation 2.1(5)]). If $\mathcal{M}$ is a model and $I \subseteq M$ is a cut, then we say that $I$ is recursively definable if there are an element $a \in M$ and two recursive sequences $t_{0}(x), t_{1}(x), t_{2}(x), \ldots$ and $t_{0}^{\prime}(x), t_{1}^{\prime}(x), t_{2}^{\prime}(x), \ldots$ of Skolem terms such that $I=\inf \left\{t_{i}(a): i<\omega\right\}=\sup \left\{t_{i}^{\prime}(a): i<\omega\right\}$. Obviously, no recursively saturated model has a recursively definable cut. In fact, $\mathcal{M}$ is recursively saturated iff it is tall and has no recursively definable cuts ([4, Theorem 2.7(i)]). There are prime models whose standard cuts are recursively definable ([5, Theorem 2.3]), and every completion of PA has a finitely generated model whose standard cut is recursively definable ([5, Coro. 2.4]).

Now, start with a nonstandard countable model $\mathcal{M}$ whose standard cut is recursively definable. By Corollary 2.5, let $\mathcal{N} \succ_{\text {cf }} \mathcal{M}$ be such that $\mathcal{N}$ is countable, $\mathcal{N} \chi^{\omega} \mathcal{M}$ and $\mathcal{N}$ has the $\omega$-property. Since the standard cut of $\mathcal{N}$ is recursively definable, $\mathcal{N}$ is not recursively saturated.

We next show that Corollary 2.5 can be somewhat improved. To do so, we need the following variant of Lemma 2.4. 
Lemma 2.6: Suppose that $\mathcal{M}$ is a countable model, $I$ is a proper cut of $\mathcal{M}$ and $X \subseteq \omega$ is such that $X \notin \operatorname{SSy}(\mathcal{M})$. If I is not uniformly $\omega$ lofty, then there is a countable $\mathcal{N} \succ_{\text {cf }} \mathcal{M}$ such that $\mathcal{N} \nsucc^{\omega} \mathcal{M}, \sup ^{\mathcal{N}}(I)$ is upward monotonically $\omega$-lofty and $X \notin \mathrm{SSy}(\mathcal{N})$.

Proof. As in the proof of Lemma 2.4, we will obtain a decreasing sequence $C_{0} \subseteq C_{1} \supseteq C_{2} \supseteq \cdots$ that has all the properties required of it in that proof. But we also need an additional property that we get by interleaving into the construction some additional steps that are applications of the following fact.

FACT 2: Suppose that $r<\omega<s \in M, I<q \in M, C$ is $\langle[r, s),[0, q)\rangle$ rich, and $g: M \longrightarrow M$ is definable. Then there are $n, v, t, u \in M$ and $\left\{d_{i}: i \in[r, t)\right\} \subseteq I$ such that $n<\omega, r \leq t<\omega<v \leq s, I<u \leq q$ and $\left\{c \in C:(g(c))_{n}=0 \Leftrightarrow n \notin X\right.$ and $(c)_{i}=d_{i}$ for $\left.r \leq i<t\right\}$ is $\langle[t, v),[0, u)\rangle$-rich.

We give a proof of this fact. Define two functions $f_{0}, f_{1}: M \times$ $[r, s] \longrightarrow[0, q]$ as follows. For each $n \in M$ and $t \in[r, s]$, let $f_{0}(n, t)$ be the largest $u \leq q$ such that $\left\{c \in C:(g(c))_{n}=0\right\}$ is $\langle[r, t),[0, u)\rangle$-rich, and let $f_{1}(n, t)$ be the largest $u \leq q$ such that $\left\{c \in C:(g(c))_{n}>0\right\}$ is $\langle[r, t),[0, u)\rangle$-rich. Both of these functions are well defined since both $\left\{c \in C:(g(c))_{n}=0\right\}$ and $\left\{c \in C:(g(c))_{n}>0\right\}$ are $\langle[r, t),[0,0)\rangle$-rich,

Observe that for each $n \in M$ and $e \in\{0,1\}$, if $r \leq t_{1} \leq t_{2} \leq s$, then $f_{e}\left(n, t_{1}\right) \geq f_{e}\left(n, t_{2}\right)$.

We consider three cases.

Case 1: There are $n<\omega$ and $v>\omega$ such that $f_{0}(n, v), f_{1}(n, v)>I$. We have two possibilities depending on whether or not $n \in X$.

(1) $n \notin X$ : Let $u=f_{0}(n, v)$. Then, $\left\{c \in C:(g(c))_{n}=0\right\}$ is $\langle[r, v),[0, u)\rangle$-rich.

(2) $n \in X$ : Let $u=f_{1}(n, v)$. Then, $\left\{c \in C:(g(c))_{n}>0\right\}$ is $\langle[r, v),[0, u)\rangle$-rich.

Case 2: There are $n<\omega$ and $t<\omega$ such that $f_{0}(n, t), f_{1}(n, t)<I$. We again have two possibilities.

(1) $n \notin X$ : Let $u=f_{1}(n, t)+1$. Since $\left\{c \in C:(g(c))_{n}>0\right\}$ is not $\langle[r, t),[0, u)\rangle$-rich, there are $d_{r}, d_{r+1}, \ldots, d_{t-1}<u$ such that there is no $c \in C$ such that $(c)_{i}=d_{i}$ whenever $r \leq i<t$ and $(g(c))_{n}>0$. Therefore, $\left\{c \in C:(g(c))_{n}=0\right.$ and $(c)_{i}=d_{i}$ for $\left.r \leq i<t\right\}$ is $\langle[t, s),[0, u)\rangle$-rich.

(2) $n \in X$ : Let $u=f_{0}(n, t)+1$. Since $\left\{c \in C:(g(c))_{n}=0\right\}$ is not $\langle[r, t),[0, u)\rangle$-rich, there are $d_{r}, d_{r+1}, \ldots, d_{t-1}<u$ such that there is no $c \in C$ such that $(c)_{i}=d_{i}$ whenever $r \leq i<t$ and $(g(c))_{n}=0$. 
Therefore, $\left\{c \in C:(g(c))_{n}>0\right.$ and $(c)_{i}=d_{i}$ for $\left.r \leq i<t\right\}$ is $\langle[t, s),[0, u)\rangle$-rich.

Case 3: Neither of the previous cases apply. Since $I$ is not uniformly $\omega$-lofty, there are $b, d$ such that $b<I<d \leq q$ and $[b, d) \cap\left\{f_{0}(n, t)\right.$ : $n<\omega, r \leq t<\omega\}=[b, d) \cap\left\{f_{1}(n, t): n<\omega, r \leq t<\omega\right\}=\varnothing$. Вy overspill, there is $u$ such that $\omega<u \leq s$ and $[b, \bar{d}) \cap\left\{f_{0}(n, t): n \leq\right.$ $u, r \leq t \leq u\}=[b, d) \cap\left\{f_{1}(n, t): n \leq u, r \leq t \leq u\right\}=\varnothing$. Without loss of generality, we can take $u=s$.

Define two functions $h_{0}, h_{1}:[0, s) \longrightarrow[0, q]$ as follows. For each $n<s$, let $h_{0}(n)=\max \left\{t \in[r, s]: f_{0}(n, t) \geq d\right\}$ and $h_{1}(n)=\max \{t \in$ $\left.[r, s]: f_{1}(n, t) \geq d\right\}$. Both functions are well defined since $f_{0}(n, r)=$ $f_{1}(n, r)=q \geq d$.

We claim, for all $n<\omega$, that $h_{0}(n)<\omega$ iff $h_{1}(n)>\omega$. For, if $h_{0}(n), h_{1}(n)>\omega$, then $v=\min \left(h_{0}(n), h_{1}(n)\right)$ would put us into Case 1. And if $h_{0}(n), h_{1}(n)<\omega$, then $t=1+\max \left(h_{0}(n), h_{1}(n)\right)$ would put us into Case 2.

Let $Y=\left\{n<\omega: h_{0}(n)<h_{1}(n)\right\}$. Clearly, $Y \in \operatorname{SSy}(\mathcal{M})$, so $Y \neq X$. Let $n<\omega$ be such that $n \in Y$ iff $n \notin X$. We have two possibilities.

(1) $n \notin X$ : Let $v=h_{0}(n)>\omega$ and continue as in (2) of Case 1 .

(2) $n \in X$ : Let $t=h_{0}(n)+1<\omega$ and then continue as in (2) of Case 2.

This completes the proof of Fact 2 .

We leave it to the reader to supply the missing details of the proof of the lemma.

Corollary 2.7: Suppose that $\mathcal{M}$ is nonstandard and countable, $X \subseteq \omega$ and $X \notin \operatorname{SSy}(\mathcal{M})$. Then there is $\mathcal{N} \succ_{\text {cf }} \mathcal{M}$ such that $\mathcal{N} \nsucc^{\omega} \mathcal{M}$, $X \notin \operatorname{SSy}(\mathcal{N})$ and $\mathcal{N}$ has the $\omega$-property.

Proof. Let $\mathcal{M}_{0}$ be a countable conservative end extension of $\mathcal{M}$. Then, one easily checks that $M$ is not a uniformly $\omega$-lofty cut of $\mathcal{M}_{0}$. Now, proceed as in the proof of Corollary 2.5 but invoking Lemma 2.6 instead of Lemma 2.4.

Both Corollaries 2.5 and 2.7 imply that some countable, nonstandard models $\mathcal{M}$ that fail to be recursively saturated have cofinal extensions $\mathcal{N}$ that have the $\omega$-property and also fail to be recursively saturated. This can happen if $\omega$ is a recursively definable cut (Corollary 2.5) or if $\operatorname{Th}(\mathcal{M}) \notin \operatorname{SSy}(\mathcal{M})$ (Corollary 2.7). We show next that, in fact, this is the case for all countable, nonstandard $\mathcal{M}$ that are not recursively saturated. 
TheOREM 2.8: Suppose that $\mathcal{M}$ is nonstandard, countable and not recursively saturated. Then there is a countable $\mathcal{N} \succ_{\mathrm{cf}} \mathcal{M}$ such that $\mathcal{N} \nsucc^{\omega} \mathcal{M}, \mathcal{N}$ has the $\omega$-property and $\mathcal{N}$ is not recursively saturated.

Proof. We can assume that $\mathcal{M}$ is tall, as otherwise Corollary 2.5 does it. Since $\mathcal{M}$ is tall and not recursively saturated, there are $a \in M$ and a recursive set $\Phi(x, y)$ of formulas such that $\Phi(x, y)$ includes $x<y$ and $\Phi(x, a)$ is finitely realizable in $\mathcal{M}$ but is omitted by $\mathcal{M}$. The cofinal extension $\mathcal{N}$ that we will obtain will fail to be recursively saturated by virtue of also omitting $\Phi(x, a)$. By [4, Lemma 2.4], there is a recursively definable cut $I$ of $\mathcal{M}$ such that for any cofinal extension $\mathcal{N}$ of $\mathcal{M}, \mathcal{N}$ realizes $\Phi(x, a)$ iff $\mathcal{N}$ fills $I$. So we will ignore trying to omit $\Phi(x, a)$ and focus instead on trying not to fill $I$.

By [5, Lemma 3.17], there is a countable $\mathcal{M}_{0} \succcurlyeq_{\text {cf }} \mathcal{M}$ that fills neither $I$ nor $\omega$ and has the property: whenever $\mathcal{N} \succ \mathcal{M}_{0}$, then $\mathcal{N}$ fills $\omega$ iff $\mathcal{N}$ fills $I^{\mathcal{M}_{0}}$. Apply Corollary 2.5 to get $\mathcal{N} \succ_{\text {cf }} \mathcal{M}_{0}$ such that $\mathcal{N} \nsucc^{\omega} \mathcal{M}_{0}$ and $\mathcal{N}$ has the $\omega$-property. Then $\mathcal{N} \chi^{\omega} \mathcal{M}$ and $\mathcal{N}$ does not fill $I^{\mathcal{M}_{0}}$, so, as an extension of $\mathcal{M}, \mathcal{N}$ does not fill $I$.

It is possible to get the natural common generalization of Lemma 2.6 and Theorem 2.8 .

Theorem 2.8 allows us to get some characterizations of countable, recursively saturated models.

Corollary 2.9: Suppose that $\mathcal{M}$ is countable and tall. The following are equivalent:

(1) $\mathcal{M}$ is recursively saturated.

(2) Every countable $\mathcal{N} \succcurlyeq_{\text {cf }} \mathcal{M}$ is generated by a set of indiscernibles.

(3) Every countable $\mathcal{N} \succcurlyeq_{\text {cf }} \mathcal{M}$ is isomorphic to some $\mathcal{N}_{0} \prec_{\text {end }} \mathcal{N}$.

Proof. The implications $(1) \Longrightarrow(2)$ and $(1) \Longrightarrow(3)$ are consequences of Proposition 1.3.

We prove the converse implications. Suppose that $\mathcal{M}$ is not recursively saturated. Let $\mathcal{N}$ be as in Theorem 2.8. By Lemma 2.2, $\mathcal{M}$ is uniformly $\omega$-lofty. Then Lemma 2.3 implies that $\mathcal{N}$ is not generated by a set of indiscernibles and there is no $\mathcal{N}_{0} \prec$ end $\mathcal{N}$ that is isomorphic to $\mathcal{N}$.

If the requirement in Corollary 2.9 that $\mathcal{M}$ is tall is replaced with the weaker one that $\mathcal{M}$ is nonstandard, then the equivalence $(1) \Longleftrightarrow(3)$ still holds. However, we don not know if $(1) \Longleftrightarrow(2)$ still holds.

We next give a characterization of those countable models that code their own theories. 
Corollary 2.10: Suppose that $T$ is a completion of PA and $\mathfrak{X}$ is a countable Scott set such that $\operatorname{Rep}(T) \subseteq \mathfrak{X}$. The following are equivalent:

(1) $T \notin \mathfrak{X}$;

(2) Whenever $\mathcal{N} \models T$ is countable and tall and $\operatorname{SSy}(\mathcal{N})=\mathfrak{X}$, then $\mathcal{N}$ has a countable, cofinal extension $\mathcal{N}_{1} \succ_{\text {cf }} \mathcal{N}$ that is not generated by a set of indiscernibles.

Proof. $\quad(1) \Longrightarrow(2)$ : Suppose that $T \notin \mathfrak{X}$. Consider an arbitrary countable and tall $\mathcal{N} \models T$ such that $\operatorname{SSy}(\mathcal{N})=\mathfrak{X}$. By Corollary 2.7, let $\mathcal{N}_{1} \succ_{\text {cf }} \mathcal{N}$ be a countable model that has the $\omega$-property such that $T \notin \operatorname{SSy}\left(\mathcal{N}_{1}\right)$. By Lemma $2.2, \mathcal{N}_{1}$ is uniformly $\omega$-lofty. Since $T=$ $\operatorname{Th}\left(\mathcal{N}_{1}\right) \notin \operatorname{SSy}\left(\mathcal{N}_{1}\right)$, then $\mathcal{N}_{1}$ is not recursively saturated, so $\mathcal{N}_{1}$ is not generated by a set of indiscernibles according to Lemma 2.3.

$(2) \Longrightarrow(1)$ : Suppose that $T \in \mathfrak{X}$. By Theorem 1.1 , we can let $\mathcal{N}$ be a countable, recursively saturated model of $T$ such that $\operatorname{SSy}(\mathcal{N})=\mathfrak{X}$. By Propositions 1.3(2) and (4), every countable $\mathcal{N}_{1} \succ_{\text {cf }} \mathcal{N}$ is generated by a set of indiscernibles.

§3. Substructure Lattices. It was proved in [13, Theorem 5.1] that if $\mathcal{M}, \mathcal{N}$ are arithmetically saturated models and $\operatorname{Lt}(\mathcal{M}) \cong \operatorname{Lt}(\mathcal{N})$, then $\operatorname{SSy}(\mathcal{M}) \cong \operatorname{SSy}(\mathcal{N})$. In this section we will take a closer look at the proof of that result in order to obtain a refinement of it stated below as Theorem 3.2. This theorem follows immediately from Lemma 3.1, which is the main result of this section and will be applied later on in proving the main results of this paper.

If $L=(L, \vee, \wedge)$ is a lattice, we will sometimes think of it as a partially ordered set $(L, \leq)$, where $x \leq y$ iff $x=x \wedge y$ iff $y=x \vee y$. We let $0_{L}$ be the least element of $L$ if there is one, and we let $1_{L}$ be the largest element if there is one. If $n<\omega$, then $\mathbf{n}$ is the lattice that is a chain having exactly $n$ elements. We let $\mathbf{B}_{2}$ be the 4-element Boolean lattice; that is, $\mathbf{B}_{2}$ is the unique 4-element lattice that is not $\mathbf{4}$. If $K, L$ are two lattices and both $1_{K}, 0_{L}$ exist, then their linear sum $K \oplus L$ is the lattice that is the disjoint union of $K$ and $L$ (except that we set $1_{K}=0_{L}$ ) such that both $K, L$ are sublattices of $K \oplus L$ and $x \leq y$ whenever $x \in K$ and $y \in L$. (For example, $\mathbf{2} \oplus \mathbf{3}=\mathbf{4}$.)

We next define some more lattices that are the same ones defined in [13, §5]. If $X \subseteq n<\omega$, we define the lattice $\mathcal{D}_{0}(X, n)$ by recursion on $n$ as follows. Let

$$
\mathcal{D}_{0}(\varnothing, 0)=\mathbf{1}
$$


and then if $X \subseteq n+1$, let

$$
\mathcal{D}_{0}(X, n+1)= \begin{cases}\mathcal{D}_{0}(X \cap n, n) \oplus \mathbf{2} & \text { if } n \in X \\ \mathcal{D}_{0}(X \cap n, n) \oplus \mathbf{B}_{2} & \text { if } n \notin X .\end{cases}
$$

Note that $\left|\mathcal{D}_{0}(X, n)\right|=3 n+1-2|X|$. (Roughly, $\mathcal{D}_{0}(X, n)$ is a stack of $n$ lattices, the $i$-th one in the stack is $\mathbf{2}$ iff $i \in X$ and is $\mathbf{B}_{2}$ iff $i \notin X$.) If $X \subseteq n+1$, then $\mathcal{D}_{0}(X \cap n, n)$ is an ideal of $\mathcal{D}_{0}(X, n+1)$. Next, if $X \subseteq n$, let $\mathcal{D}(X, n)=\mathcal{D}_{0}(X, n) \oplus \mathbf{2}$. If $X \subseteq n+1$, we consider that $\mathcal{D}(X \cap n, n) \subseteq \mathcal{D}(X, n+1)$ by setting $1_{\mathcal{D}(X \cap n, n)}=1_{\mathcal{D}(X, n+1)}$.

If $X \subseteq \omega$, we define $\mathcal{D}(X)=\bigcup_{n<\omega}\left(\mathcal{D}(X \cap n, n)\right.$, and then let $\mathcal{D}^{\prime}(X)$ be the lattice obtained from $\mathcal{D}(X)$ by adding one more element that is less than $1_{\mathcal{D}(X)}$ but greater than every other element of $\mathcal{D}(X)$. Thus, each $\mathcal{D}(X \cap n, n)$ is a sublattice of $\mathcal{D}(X)$ which, in turn, is a sublattice of $\mathcal{D}^{\prime}(X)$. In fact, $\mathcal{D}^{\prime}(X)$ is the completion of $\mathcal{D}(X)$, and $\mathcal{D}(X)$ is the sublattice of $\mathcal{D}^{\prime}(X)$ consisting of the compact elements in $\mathcal{D}^{\prime}(X)$.

Lemma 3.1: Suppose that $\mathcal{M}$ is recursively saturated and $X \subseteq \omega$. The following are equivalent.

(1) There is $\mathcal{M}_{1} \prec \mathcal{M}$ such that $\operatorname{Lt}\left(\mathcal{M}_{1}\right) \cong \mathcal{D}^{\prime}(X)$.

(2) There is $Y \in \operatorname{SSy}(\mathcal{M})$ such that $X \leq_{T} Y^{\prime}$.

Proof. $(1) \Longrightarrow(2)$ : Let $\mathcal{M}_{1} \prec \mathcal{M}$ be such that $\operatorname{Lt}\left(\mathcal{M}_{1}\right) \cong \mathcal{D}^{\prime}(X)$. Since $1_{\mathcal{D}^{\prime}(X)}$ exists and has a unique immediate predecessor, it must be that $\mathcal{M}_{1}$ is finitely generated. Let $\mathcal{M}_{1}=\operatorname{Scl}(a)$, and $Y=\operatorname{tp}(a)$. Since, $\mathcal{M}$ is recursively saturated, $Y \in \operatorname{SSy}(\mathcal{M})$.

We easily see that $X$ is $\Delta_{2}^{0}$ in $Y$. First, $n \in X$ iff there is $Z \subseteq n+1$ such that $n \in Z$ and there are Skolem terms $t_{r}(x)$ for each $r \in \mathcal{D}_{0}(Z, n)$ (where we let $m=1_{\mathcal{D}_{0}(z, n)}$ and also Skolem terms $t_{r s}(y)$ for each $r \leq$ $s \in L(Z, n)$ such that each formula $t_{r s}\left(t_{s}(x)\right)=t_{r}(x)$ is in $\operatorname{tp}(a)$ and whenever $t(y)$ is a Skolem term, then for each $s \in \mathcal{D}_{0}(Z, n)$ there is a unique $r \leq s$ such that $t\left(t_{s}(x)\right)=t_{r}(x)$ is in $\operatorname{tp}(a)$. Similarly, $n \notin X$ iff there is $Z \subseteq n+1$ such that $n \notin Z$ and all the same conditions hold for this $Z$.

$(2) \Longrightarrow(1)$ : This part of the proof relies on the technology for constructing models with a prescribed substructure lattice as presented in [15. Chap. 4.5]. Since the application of this technology is quite routine, we will present just a sketch of the proof.

Let $\mathcal{M}_{0}$ be the prime elementary submodel of $\mathcal{M}$. For some finite lattices $L$, we define by recursion a representation $\alpha_{L}: L \longrightarrow \operatorname{Eq}(A)$, which we will call a regular representation. Each regular representation 
that we define will be definable in $\mathcal{M}_{0}$, and we will define it only up to $\mathcal{M}_{0}$-definable isomorphism.

- If $L=\mathbf{2}$, then $\alpha_{L}$ is regular if $A=M_{0}$.

- If $L=\mathbf{B}_{2}$, then assume that $b_{1}, b_{2}$ are the two atoms of $\mathbf{B}_{2}$. Then, $\alpha_{L}$ is regular if $A=\left\{\left\langle x_{1}, x_{2}\right\rangle \in M_{0}^{2}: x_{1}<x_{2}\right\}$, and whenever $\left\langle x_{1}, x_{2}\right\rangle,\left\langle y_{1}, y_{2}\right\rangle \in A$ and $e \in\{1,2\}$, then $\left\langle\left\langle x_{1}, x_{2}\right\rangle\right.$, $\left.\left\langle y_{1}, y_{2}\right\rangle\right\rangle \in \alpha\left(b_{e}\right)$ iff $x_{e}=y_{e}$.

- If $\alpha_{0}: L_{0} \longrightarrow \operatorname{Eq}\left(A_{0}\right)$ and $\alpha_{1}: L_{1} \longrightarrow \operatorname{Eq}\left(A_{1}\right)$ are regular and $L=L_{0} \oplus L_{1}$, then $\alpha: L \longrightarrow \operatorname{Eq}(A)$ is regular if $A=$ $A_{0} \times A_{1}$ and whenever $\left\langle x_{0}, x_{1}\right\rangle,\left\langle y_{0}, y_{1}\right\rangle \in A$ and $r \in L_{0} \oplus L_{1}$, then $\left\langle\left\langle x_{0}, x_{1}\right\rangle,\left\langle y_{0}, y_{1}\right\rangle\right\rangle \in \alpha(r)$ iff one of the following holds:

(0) $r \in L_{0}$ and $\left\langle x_{0}, y_{0}\right\rangle \in \alpha_{0}(r)$,

(1) $r \in L_{1}, x_{0}=y_{0}$ and $\left\langle x_{1}, y_{1}\right\rangle \in \alpha_{1}(r)$.

Whenever $L=\mathcal{D}_{0}(X, n)$ or $L=\mathcal{D}(X, n)$, the regular representation is well defined and unique (up to $\mathcal{M}$-definable isomorphism).

We observe some facts about regular representations of the $\mathcal{D}(X, n)$ 's. Suppose that $\alpha: \mathcal{D}(X, n) \longrightarrow \operatorname{Eq}(A)$ is a regular representation.

(1) If $m<n$, then $\alpha / \mathcal{D}(X \cap m, m)$ is regular.

(2) If $n<m<\omega, Y \subseteq m$ and $X=Y \cap n$, then there a regular representation $\beta: \mathcal{D}(Y, m) \longrightarrow \operatorname{Eq}(A)$ such that $\alpha=\beta \mid \mathcal{D}(X, n)$.

(3) If $\Theta \in \operatorname{Eq}(A)$ is definable in $\mathcal{M}_{0}$, then there is $B \subseteq A$ such that $\alpha \mid B$ is regular and there is $r \in \mathcal{D}(X, n)$ such that $\alpha(r) \cap B^{2}=$ $\Theta \cap B^{2}$.

The proofs of $(1)-(3)$ will be omitted. The reader can consult with [13, §5] where some analogous statements are proved.

Each of (1) - (3) is effective. This is trivial for (1). For (2), this means that given $n<m<\omega, Y \subseteq m$ and a formula that defines $\alpha$, then a formula that defines $\beta$ can be effectively obtained. For (3), this means that given $n<\omega$ and formulas that define $\alpha$ and $\Theta$, then a formula defining $B$ can be effectively obtained.

Since $\operatorname{Th}(\mathcal{M}) \in \operatorname{SSy}(\mathcal{M})$, we can assume without loss of generality that $\operatorname{Th}(\mathcal{M}) \leq_{T} Y$.

Let $\left\langle X_{n}: n<\omega\right\rangle$ be a sequence of finite sets that is recursive in $Y^{\prime}$ such that $\lim _{n} X_{n}=X$ in the sense that for all $i<\omega$, there is $m<\omega$ such that whenever $m \leq n<\omega$, then $i \in X$ iff $i \in X_{n}$. We can also require that there is a sequence $0=k_{0}, k_{1}, k_{2}, \ldots$ such that whenever $n<\omega$, then $X_{n} \subseteq k_{n}<\omega$ and either $k_{n+1}=k_{n}+1$ and $X_{n}=X_{n+1} \cap n$ or else $k_{n+1} \leq k_{n}$ and $X_{n+1}=X_{n} \cap n+1$.

Let $\theta_{0}(x, y), \theta_{1}(x, y), \theta_{2}(x, y), \ldots$ be a recursive list of 2-ary formulas in the language of PA so that each definable equivalence relation $\Theta \subseteq$ 
$M_{0}^{2}$ is defined by infinitely many of the formulas. We construct a sequence of regular representations $\left\langle\alpha_{n}: \mathcal{D}\left(X_{n}, k_{n}\right) \longrightarrow A_{n}\right\rangle$. (More precisely, we construct a sequence $\varphi_{0}, \varphi_{1}, \varphi_{2}, \ldots$ of formulas such that $\varphi_{n}$ defines $\alpha_{n}$ in $\mathcal{M}_{0}$, and this sequence should be recursive in $Y^{\prime}$.)

Let $\alpha_{0}: \mathbf{2} \longrightarrow \operatorname{Eq}\left(M_{0}\right)$ be a regular representation. Now suppose that we $\alpha_{n}$. We will effectively obtain $\alpha_{n+1}$. If $k_{n+1}=k_{n}+1$, apply (2) to get a regular $\alpha: \mathcal{D}\left(X_{n+1}, k_{n+1}\right.$, and if $k_{n+1} \leq k_{n}$, then let $\alpha=\alpha_{n} \backslash \mathcal{D}\left(X_{n+1}, k_{n+1}\right)$. Since $\alpha$ is regular, we apply $(3)$ with $\Theta$ being the equivalence relation defined bt $\theta_{n}$ to get $\alpha_{n+1}=\alpha \mid B$.

Thus, for each $n<\omega$, we have $\alpha_{n}: L\left(X_{n}, k_{n}\right) \longrightarrow \operatorname{Eq}\left(A_{n}\right)$. The sequence $A_{0} \supseteq A_{1} \supseteq A_{2} \supseteq$ is recursive and determines a complete type $p(x)$. Let $a$ realize this type in $\mathcal{M}$, and then let $\mathcal{M}_{1}=\operatorname{Scl}(a)$.

One then checks that $\operatorname{Lt}\left(\mathcal{M}_{1}\right) \cong \mathcal{D}^{\prime}(X)$.

The previous lemma easily implies the following theorem.

THEOREM 3.2: If $\mathcal{M}, \mathcal{N}$ are recursively saturated models and $\operatorname{Lt}(\mathcal{M})$ $\cong \operatorname{Lt}(\mathcal{N})$, then for each $X \in \operatorname{SSy}(\mathcal{M})$ there is $Y \in \operatorname{SSy}(\mathcal{N})$ such that $X^{\prime} \equiv_{T} Y^{\prime}$.

If $\mathcal{M}$ is recursively saturated, then $\mathbf{M}_{3}$ is an ideal of $\operatorname{Lt}(\mathcal{M})$ iff $\mathcal{M}$ is not a model of TA. Can Theorem 3.2 be improved if neither (or both) are models of TA?

§4. The Proof of Theorem 4. Lascar's Theorem shows (in the terminology of [19]) that open subgroups are recognizable for the class of countable, arithmetically saturated models. This means: if $\mathcal{M}, \mathcal{N}$ are countable, arithmetically saturated models, $\alpha: \operatorname{Aut}(\mathcal{M}) \longrightarrow \operatorname{Aut}(\mathcal{N})$ is an isomorphism and $H \leq \operatorname{Aut}(\mathcal{M})$, then $H$ is an open subgroup of $\operatorname{Aut}(\mathcal{M})$ iff $\alpha[H]$ is an open subgroup of $\operatorname{Aut}(\mathcal{N})$. This consequence of Lascar's Theorem was improved in [19, Coro. 3.14] where it was shown that basic open subgroups are recognizable 1 In particular, if $\mathcal{M}, \mathcal{N}$ are countable, arithmetically saturated models, $\alpha: \operatorname{Aut}(\mathcal{M}) \longrightarrow \operatorname{Aut}(\mathcal{N})$ is an isomorphism and $H \leq \operatorname{Aut}(\mathcal{M})$, then $H$ is the stabilizer of a finite set iff $\alpha[H]$ (as a subgroup of $\operatorname{Aut}(\mathcal{N})$ ) is the stabilizer of a finite set. This allows us to define the function $\widetilde{\alpha}: \operatorname{Lt}_{0}(\mathcal{M}) \longrightarrow \operatorname{Lt}_{0}(\mathcal{N})$ as follows: If $\mathcal{M}_{1} \in \operatorname{Lt}_{0}(\mathcal{M})$, then $\widetilde{\alpha}\left(\mathcal{M}_{1}\right)$ is that unique $\mathcal{N}_{1} \in \operatorname{Lt}_{0}(\mathcal{N})$ such that $\operatorname{Aut}(\mathcal{N})_{\left(N_{1}\right)}=\alpha\left[\operatorname{Aut}(\mathcal{M})_{\left(M_{1}\right)}\right]$. We easily see that $\widetilde{\alpha}$ is an isomorphism from the semilattice $\operatorname{Lt}_{0}(\mathcal{M})$ onto the semilattice $\operatorname{Lt}_{0}(\mathcal{N})$. Thus, it extends uniquely to an isomorphism of the lattices $\operatorname{Lt}(\mathcal{M})$

\footnotetext{
${ }^{1}$ Henceforth, the term "recognizable" will be used in a rather informal way, and it is be understood as meaning "recognizable for the class of arithmetically saturated models".
} 
and $\operatorname{Lt}(\mathcal{N})$. We denote this extension also by $\widetilde{\alpha}$. Thus, we have the following lemma.

Lemma 4.1: Suppose that $\mathcal{M}, \mathcal{N}$ are countable, arithmetically saturated models and that $\alpha: \operatorname{Aut}(\mathcal{M}) \longrightarrow \operatorname{Aut}(\mathcal{N})$ is an isomorphism. Then $\widetilde{\alpha}: \operatorname{Lt}(\mathcal{M}) \longrightarrow \operatorname{Lt}(\mathcal{N})$ is an isomorphism.

We will typically invoke this lemma without referencing it.

The isomorphism $\widetilde{\alpha}$ is implicit in [19, Coro. 3.15]. Observe that if $a \in M$ and $g \in \operatorname{Aut}(\mathcal{M})$, then $g \in \operatorname{Aut}(\mathcal{M})_{a}$ iff $\alpha(g) \in \widetilde{\alpha}\left(\operatorname{Aut}(\mathcal{M})_{a}\right)$.

The map $\alpha \mapsto \widetilde{\alpha}$ is functorial in the sense that if $\mathcal{M}_{1}, \mathcal{M}_{2}, \mathcal{M}_{3}$ are countable, arithmetically saturated models and $\alpha: \operatorname{Aut}\left(\mathcal{M}_{1}\right) \longrightarrow$ $\operatorname{Aut}\left(\mathcal{M}_{2}\right), \beta: \operatorname{Aut}\left(\mathcal{M}_{2}\right) \longrightarrow \operatorname{Aut}\left(\mathcal{M}_{3}\right)$ are isomorphisms, then $\widetilde{\beta \alpha}=$ $\widetilde{\beta} \widetilde{\alpha}$ and $\widetilde{\alpha^{-1}}=\widetilde{\alpha}^{-1}$.

We can now comment about the general strategy that is used in this section. Of course, the ultimate goal is to prove Theorem 4. It will be seen that Theorem 4 follows almost immediately from Lemma 3.1 and the fact, to be proved as Lemma 4.15, that recursively saturated structures are recognizable. To get that conclusion, we will prove that recursive saturation can be characterized in terms of properties already shown to be recognizable. But to get the recognizability of these other properties, we will show that they too are characterizable in terms of other properties that were previously shown to be recognizable. And so on. Thus, we will build a catalogue of recognizable properties and show that recursive saturation is in this catalogue.

We begin by showing that isomorphism is recognizable

Lemma 4.2: Suppose that $\mathcal{M}, \mathcal{N}$ are countable, arithmetically saturated models and that $\alpha: \operatorname{Aut}(\mathcal{M}) \longrightarrow \operatorname{Aut}(\mathcal{N})$ is an isomorphism. If $\mathcal{M}_{1}, \mathcal{M}_{2} \preccurlyeq \mathcal{M}$ and $\mathcal{M}_{1} \cong \mathcal{M}_{2}$, then $\widetilde{\alpha}\left(\mathcal{M}_{1}\right) \cong \widetilde{\alpha}\left(\mathcal{M}_{2}\right)$.

Proof. Let $G=\operatorname{Aut}(\mathcal{M})$. Suppose that $\mathcal{M}_{1}, \mathcal{M}_{2} \preccurlyeq \mathcal{M}$ and that $\mathcal{M}_{1} \cong \mathcal{M}_{2}$. Let $\mathcal{N}_{1}=\widetilde{\alpha}\left(\mathcal{M}_{1}\right)$ and $\mathcal{N}_{2}=\widetilde{\alpha}\left(\mathcal{M}_{2}\right)$. We wish to show that $\mathcal{N}_{1} \cong \mathcal{N}_{2}$.

First, suppose that $\mathcal{M}_{1}$ is finitely generated. Then, $\mathcal{M}_{2}$ is finitely generated, and $G_{\left(M_{1}\right)}$ and $G_{\left(M_{2}\right)}$ are conjugate subgroups, so let $g \in G$ be such that $g G_{\left(M_{1}\right)} g^{-1}=G_{\left(M_{2}\right)}$. Applying $\alpha$ yields

$$
\alpha(g) \operatorname{Aut}(\mathcal{N})_{\left(N_{1}\right)}(\alpha(g))^{-1}=\operatorname{Aut}(\mathcal{N})_{\left(N_{2}\right)},
$$

so that $\operatorname{Aut}(\mathcal{N})_{\left(N_{1}\right)}, \operatorname{Aut}(\mathcal{N})_{\left(N_{2}\right)}$ are conjugate subgroups of $\operatorname{Aut}(\mathcal{N})$. Therefore, $\mathcal{N}_{1} \cong \mathcal{N}_{2}$. 
We have just proved the lemma in the case $\mathcal{M}_{1}, \mathcal{M}_{2}$ are finitely generated. Observe that if $f \in G$, then $f\left\lceil M_{1}\right.$ is an isomorphism from $\mathcal{M}_{1}$ onto $\mathcal{M}_{2}$ iff $f G_{\left(M_{1}\right)} f^{-1}=G_{\left(M_{2}\right)}$.

Next, suppose that $\mathcal{M}_{1} \preccurlyeq \mathcal{M}$ is not finitely generated, and that $\varphi: \mathcal{M}_{1} \longrightarrow \mathcal{M}_{2}$ is an isomorphism. Let $\mathcal{M}_{1,0} \prec \mathcal{M}_{1,1} \prec \mathcal{M}_{1,2} \prec \cdots$ be a sequence of finitely generated, elementary substructures of $\mathcal{M}_{1}$ such that $\mathcal{M}_{1}=\bigcup_{i<\omega} \mathcal{M}_{1, i}$. For each $i<\omega$, let $\varphi_{i}=\varphi\left\lceil M_{0, i}\right.$ and $\mathcal{M}_{2, i}=\varphi_{i}\left[\mathcal{M}_{1, i}\right]$. Then, $\mathcal{M}_{2}=\bigcup_{i<\omega} \mathcal{M}_{2, i}$. Let $f_{i} \in G$ be such that $f_{i} \supseteq \varphi_{i}$. Since $\varphi_{0} \subseteq \varphi_{1} \subseteq \varphi_{2} \subseteq \cdots$, we have that whenever $i \leq j<\omega$, then $f_{j} G_{\left(M_{0, i}\right)} f^{-1}=G_{\left(M_{1, i}\right)}$.

Let $\mathcal{N}_{1, i}=\widetilde{\alpha}\left(\mathcal{M}_{1, i}\right)$ and $\mathcal{N}_{2, i}=\widetilde{\alpha}\left(\mathcal{M}_{2, i}\right.$. Then, $\mathcal{N}_{1}=\bigcup_{i<\omega} \mathcal{N}_{1, i}$ and $\mathcal{N}_{2}=\bigcup_{i<\omega} \mathcal{N}_{2, i}$. By the first part of this proof, each $\mathcal{N}_{0, i} \cong \mathcal{N}_{1, i}$. Let $\theta_{i}: \mathcal{N}_{0, i} \longrightarrow \mathcal{N}_{1, i}$ be the unique isomorphism. Let $g_{i}=\alpha\left(f_{i}\right)$. Then, whenever $i \leq j<\omega$, then $g_{j} \operatorname{Aut}(\mathcal{N})_{\left(N_{0, i}\right)} g_{j}^{-1}=\operatorname{Aut}(\mathcal{N})_{\left(N_{1, i}\right)}$. Thus, if $i \leq j<\omega$, then $g_{j}\left\lceil N_{0, i}: \mathcal{N}_{0, i} \longrightarrow \mathcal{N}_{1, i}\right.$ is an isomorphism. Hence, $\bigcup_{i<\omega} g_{i}\left\lceil N_{0, i}\right.$ is an isomorphism from $\mathcal{N}_{0}$ onto $\mathcal{N}_{1}$.

The next lemma says that both cofinal extensions and end extensions are recognizable and also that both tall models and short models are recognizable.

Lemma 4.3: Suppose that $\mathcal{M}, \mathcal{N}$ are countable, arithmetically saturated models, $\alpha: \operatorname{Aut}(\mathcal{M}) \longrightarrow \operatorname{Aut}(\mathcal{N})$ is an isomorphism and $\mathcal{M}_{1}, \mathcal{M}_{2}$ $\preccurlyeq \mathcal{M}$.

(1) If $\mathcal{M}_{1} \prec_{\text {cf }} \mathcal{M}_{2}$, then $\widetilde{\alpha}\left(\mathcal{M}_{1}\right) \prec_{\text {cf }} \widetilde{\alpha}\left(\mathcal{M}_{2}\right)$.

(2) If $\mathcal{M}_{1} \prec_{\text {end }} \mathcal{M}_{2}$, then $\widetilde{\alpha}\left(\mathcal{M}_{1}\right) \prec_{\text {end }} \widetilde{\alpha}\left(\mathcal{M}_{2}\right)$.

(3) If $\mathcal{M}_{1}$ is tall, then $\widetilde{\alpha}\left(\mathcal{M}_{1}\right)$ is tall.

(4) If $\mathcal{M}_{1}$ is short, then $\widetilde{\alpha}\left(\mathcal{M}_{1}\right)$ is short.

Proof. First, note that the special case of (2) in which $\mathcal{M}_{2}=\mathcal{M}$ is one of the parts of [19, Prop. 2.1] and is also [15, Coro. 9.4.10], where a proof is given. For us, an easier proof comes from an application of Kaye's Theorem, or, more precisely, the *-version of Kaye's Theorem that applies to structures of the form $(\mathcal{M}, a)$, where $a \in M$. Observe that $H<\operatorname{Aut}(\mathcal{M})$ is the pointwise stabilizer of a short elementary cut iff for some $a \in M, H$ is the smallest, nontrivial, closed normal subgroup of $\operatorname{Aut}(\mathcal{M})_{a}=\operatorname{Aut}(M, a)$. Then note that every elementary cut is the union of a set of short elementary cuts, and, conversely, the union of any set of short elementary cuts is an elementary cut.

We now prove $(1)-(4)$.

(1): This is due to the following equivalence: $\mathcal{M}_{1} \prec_{\text {cf }} \mathcal{M}_{2}$ iff $\mathcal{M}_{1} \prec$ $\mathcal{M}_{2}$ and whenever $\mathcal{M}_{1} \preccurlyeq \mathcal{M}_{3} \prec$ end $\mathcal{M}$, then $\mathcal{M}_{2} \preccurlyeq \mathcal{M}_{3}$. 
(2): This is a consequence of (1), and the following equivalence: $\mathcal{M}_{1} \prec$ end $\mathcal{M}_{2}$ iff $\mathcal{M}_{1} \prec \mathcal{M}_{2}$ and there is no $\mathcal{M}_{3}$ such that $\mathcal{M}_{1} \prec_{\text {cf }}$ $\mathcal{M}_{3} \preccurlyeq \mathcal{M}_{2}$.

(3): This is a consequence of (2) since $\mathcal{M}_{1}$ is tall iff there is a sequence $\mathcal{M}_{1,0} \prec_{\text {end }} \mathcal{M}_{1,1} \prec_{\text {end }} \mathcal{M}_{1,2} \prec_{\text {end }} \cdots$ such that $\mathcal{M}_{1}=\bigcup_{i<\omega} \mathcal{M}_{1, i}$.

(3): $\mathcal{M}_{1}$ is short iff $\mathcal{M}_{1}$ is not tall.

Suppose that $\mathcal{M}$ is arithmetically saturated. The interstices and interstitial gaps of $\mathcal{M}$ were first defined and studied in [2]. The least interstice of $\mathcal{M}$, denoted by $\Omega_{\omega}$, is the set $\{x \in M: \omega<x<a$ for all $a \in \operatorname{Scl}(\varnothing) \backslash \omega\}$. The arithmetic saturation of $\mathcal{M}$ entails that $\Omega_{\omega} \neq \varnothing$. Observe that $\Omega_{\omega} \cup \omega$ is the smallest nonstandard invariant cut of $\mathcal{M}$. We partition $\Omega_{\omega}$ into convex sets, called interstitial gaps (or igaps for short) as follows. First, let $\mathcal{F}$ be the set of $\varnothing$-definable functions $f$ : $M \longrightarrow M$ such that whenever $x \leq y<\omega$, then $x \leq f(x) \leq f(y)<\omega$. Then, for each $a \in \Omega_{\omega}$, define the igap around $a$ to be the set

$$
\operatorname{igap}(a)=\left\{b \in \Omega_{\omega}: a \leq f(b) \text { and } b \leq f(a) \text { for some } f \in F^{\mathcal{M}}\right\} .
$$

The set of igaps is linearly ordered with order type of the rationals. It is a routine exercise in recursive saturation to show that whenever $\gamma_{1}<\gamma_{2}$ are igaps and $a \in \Omega_{\omega}$, then there is $g \in \operatorname{Aut}(\mathcal{M})$ such that $\gamma_{1}<g(a)<\gamma_{2}$. A cut $I \subseteq M$ is an icut if $I \subseteq \omega \cup \Omega_{\omega}$ and whenever $\gamma$ is an igap such that $\gamma \cap I \neq \varnothing$, then $\gamma \subseteq I$.

Kaye's Theorem implies that $\operatorname{Aut}(\mathcal{M})_{\left(\Omega_{\omega}\right)}$ is the largest, closed proper normal subgroup of $\operatorname{Aut}(\mathcal{M})$.

The following lemma improves Proposition 4.2 of [19] by eliminating the hypothesis that both $\mathcal{M}$ and $\mathcal{N}$ are 2-Ramsey 2

Lemma 4.4: Suppose that $\mathcal{M}, \mathcal{N}$ are countable, arithmetically saturated models and that $\alpha: \operatorname{Aut}(\mathcal{M}) \longrightarrow \operatorname{Aut}(\mathcal{N})$ is an isomorphism. Suppose that $H_{1} \leq \operatorname{Aut}(\mathcal{M})$ and $H_{2}=\alpha\left[H_{1}\right] \leq \operatorname{Aut}(\mathcal{N})$.

(a) If $H_{1}$ is a pointwise stabilizer of an icut, then so is $\mathrm{H}_{2}$.

(b) If $H_{1}$ is a setwise stabilizer of an icut, then so is $H_{2}$.

(c) If $\mathrm{H}_{1}$ is a pointwise stabilizer of an igap, then so is $\mathrm{H}_{2}$.

(d) If $\mathrm{H}_{1}$ is a setwise stabilizer of an igap, then so is $\mathrm{H}_{2}$.

Proof. We will prove (a). Then parts (b) - (d) will follow just as in the proof of [19, Prop. 4.2].

Let $G=\operatorname{Aut}(\mathcal{M})$. The concepts of least interstice, igap and icut extend naturally to models $(\mathcal{M}, a)$, where $a \in M$.

\footnotetext{
${ }^{2}$ According to [19], a model $\mathcal{M}$ is $n$-Ramsey iff $(\mathbb{N}, \operatorname{Rep}(\operatorname{Th}(\mathcal{M}))) \models \mathrm{RT}_{2}^{n}$.
} 
Suppose that $a \in M$. Let $J(a)=\sup \left(\left(\omega \cup \Omega_{\omega}\right) \cap \operatorname{Scl}(a)\right)$ and then let $N(a)=G_{(J(a))} \cap G_{a}$. It is easily checked that $J(a)$ is an icut. By the *-version of Kaye's Theorem (applied to $(\mathcal{M}, a)$ ), $N(a)$ is the smallest closed subgroup $H$ such that $G_{\left(\Omega_{\omega}\right)} \cap G_{a}<H \unlhd G_{a}$.

We will say that two groups $N(a)$ and $N(b)$ are equivalent if there is $c \in M$ such that $N(a) \cap G_{c}=N(b) \cap G_{c}$.

Claim: If $a, b \in M$, then $N(a)$ and $N(b)$ are equivalent iff $J(a)=$ $J(b)$.

To prove the claim, consider $a, b \in M$. If $J(a)=J(b)$, then $c=\langle a, b\rangle$ is such that $N(a) \cap G_{c}=N(b) \cap G_{c}$.

For the converse, suppose that $J(b) \backslash J(a) \neq \varnothing$. Then, by recursive saturation, for any $c \in M$, there is $f \in G_{(J(a)} \cap G_{c}$ that moves some $d \in J(b)$. This proves the claim.

It follows from the arithmetic saturation of $\mathcal{M}$ that for any $c \in$ $M \backslash \operatorname{Scl}(J(a))$, there is an $f \in G_{(J(a))}$ such that $f(c) \neq c$. Thus, we have that $G_{(J(a))}$ is the closure of $\bigcup\{N(b): N(b)$ is equivalent to $N(a)\}$. Thus, for any $a \in M$ there is $b \in N$ such that $\widetilde{\alpha}(\operatorname{Scl}(N(a))=\operatorname{Scl}(N(b))$.

Now suppose that $I$ is an arbitrary icut. Either there is an igap such that $I=\sup (\gamma)$ or there is not. If there is no such $\gamma$, then $I$ is the union of all those $J(a)$ such that $J(a) \subseteq I$, and if there is such a $\gamma$, then $I$ is the intersection of all those $J(a)$ such that $J(a) \supseteq I$. Thus, $H$ is the pointwise stabilizer of an icut iff it is either the union or the intersection of a set of subgroups of the form $G_{(J(a))}$, from which (a) easily follows.

In the next lemma, we formally record that certain kinds of models are recognizable. In particular, those described in (2) and (3) of Lemma 2.3 are. The recognizability of those models in (1) of Lemma 2.3 will be shown in Lemma 4.15.

Lemma 4.5: Suppose that $\mathcal{M}, \mathcal{N}$ are countable, arithmetically saturated models and that $\alpha: \operatorname{Aut}(\mathcal{M}) \longrightarrow \operatorname{Aut}(\mathcal{N})$ is an isomorphism. Suppose that $\mathcal{M}_{0} \prec \mathcal{M}$.

(1) If $\mathcal{M}_{0}$ is homogeneous, then $\widetilde{\alpha}\left(\mathcal{M}_{0}\right)$ is homogeneous.

(2) If $\mathcal{M}_{0}$ is generated by a set of indiscernibles, then so is $\widetilde{\alpha}\left(\mathcal{M}_{0}\right)$.

(3) If $\mathcal{M}_{0}$ is isomorphic to some $\mathcal{M}_{1} \prec$ end $\mathcal{M}_{0}$, then $\widetilde{\alpha}\left(\mathcal{M}_{0}\right)$ is isomorphic to some $\mathcal{N}_{1} \prec$ end $\widetilde{\alpha}\left(\mathcal{M}_{0}\right)$.

Proof. The proofs are straightforward. We only note that (1) follows from the characterization: $\mathcal{M}_{0}$ is homogeneous iff whenever $a_{0}, a_{1}, b_{0} \in$ $M_{0}$ and $\operatorname{tp}\left(a_{0}\right)=\operatorname{tp}\left(b_{0}\right)$, then there is $b_{1} \in M_{0}$ such that $\operatorname{tp}\left(a_{0}, a_{1}\right)=$ $\operatorname{tp}\left(b_{0}, b_{1}\right)$. 
One sort of problem that arises when working inside an arithmetically saturated model $\mathcal{M}$ is that some familiar constructions cannot be carried out. For an example, take the MacDowell-Specker Theorem: For every $\mathcal{M}_{0}$ there is $\mathcal{M}_{1}$ such that $\mathcal{M}_{0} \prec$ end $\mathcal{M}_{1}$. However, even if $\mathcal{M}$ is arithmetically saturated, there is $\mathcal{M}_{0} \prec \mathcal{M}$ for which there is no $\mathcal{M}_{1}$ such that $\mathcal{M}_{0} \prec$ end $\mathcal{M}_{1} \preccurlyeq \mathcal{M}$. One way around this problem is by restricting attention to just the coded elementary substructures of $\mathcal{M}$. We next make the appropriate definitions.

If $\mathcal{M}$ is a model, then a subset $A \subseteq M$ is coded $($ in $\mathcal{M})$ if there is $a \in M$ such that $A=\left\{(a)_{i}: i<\omega\right\}$. In particular, $\operatorname{SSy}(\mathcal{M})$ is the set of coded subsets of $\omega$. A model $\mathcal{M}_{0}$ is coded in $\mathcal{M}$ if $\mathcal{M}_{0} \prec \mathcal{M}$ and $M_{0}$ is coded. If $\mathcal{M}$ is recursively saturated and $A \subseteq M$ is coded, then $\operatorname{Scl}(A)$ is coded. Clearly, every finitely generated elementary substructure of a recursively saturated model is coded. If $\mathcal{M}$ is recursively saturated and $\mathcal{M}_{0} \prec \mathcal{M}$ is coded, then there is a coded $\mathcal{M}_{1} \prec \mathcal{M}$ such that $\mathcal{M}_{0} \prec$ end $\mathcal{M}_{1}$. The following proposition shows that even more is true.

Proposition 4.6: Suppose that $\mathcal{M}$ is recursively saturated and $\mathcal{M}_{0} \prec \mathcal{M}$ is coded. Then there is a coded, tall $\mathcal{M}_{1} \prec \mathcal{M}$ such that $\mathcal{M}_{0} \prec$ end $\mathcal{M}_{1}$ and $\mathcal{M}_{1}$ is a conservative extension of $\mathcal{M}_{0}$.

Proof. Suppose that $\mathcal{M}_{0}$ is coded. Let $a \in M$ realize a minimal type in $\mathcal{M}$. By recursive saturation, there is $b \in M$ such that for each $i<\omega$, $M_{0}<(b)_{i}<(b)_{i+1}$ and $\operatorname{tp}\left((b)_{i}\right)=\operatorname{tp}(a)$. Then $\mathcal{M}_{1}=\operatorname{Scl}\left(M_{0} \cup\left\{(b)_{i}\right.\right.$ : $i<\omega\})$ is coded and tall and is a conservative extension of $\mathcal{M}_{0}$.

Recall the notion of a superminimal extension as in [15, §2.1.2]. If $\mathcal{M}_{1} \prec \mathcal{M}_{2}$, then $\mathcal{M}_{2}$ is a superminimal extension of $\mathcal{M}_{1}$ if $\mathcal{M}_{3} \preccurlyeq \mathcal{M}_{1}$ for every $\mathcal{M}_{3} \prec \mathcal{M}_{2}$. It is clear that superminimal extensions are recognizable.

Lemma 4.7: Suppose that $\mathcal{M}$ is arithmetically saturated and $\mathcal{M}_{1} \preccurlyeq$ $\mathcal{M}$. Then $\mathcal{M}_{1}$ is coded iff $\mathcal{M}_{1}$ has a superminimal end extension $\mathcal{M}_{2} \prec$ $\mathcal{M}$.

Proof. $(\Longrightarrow)$ : Every countable model has a superminimal elementary end extension. After checking the proof of this (for example, in [15, Theorem 2.1.12]), we see that if $\mathcal{M}_{1}$ is coded, then a superminimal extension $\mathcal{M}_{2}$ can be constructed so that $\mathcal{M}_{2} \prec \mathcal{M}$.

$(\Longrightarrow)$ : Let $\mathcal{M}_{2} \prec \mathcal{M}$ be a superminimal extension of $\mathcal{M}_{1}$. (It is not necessary that it be an end extension.) First note that $\mathcal{M}_{2}$ is finitely generated, so it is coded. Let $b \in M$ code $M_{2}$; that is, $M_{2}=\left\{(b)_{n}: n<\right.$ $\omega\}$. Let $t_{0}(x), t_{1}(x), t_{2}(x), \ldots$ be a recursive list of all Skolem terms. 
Let $I \subseteq \omega$ be such that $i \in I$ iff $t_{i}(b) \in M_{1}$. Thus, $i \in I$ iff there is no Skolem term $t(y)$ such that $t\left(t_{i}(b)\right)=b$. Clearly, $I$ is recursive in $\operatorname{tp}(b)^{\prime}$. Let $i_{n}$ be the $n$-th member of $I$. Since $\mathcal{M}$ is arithmetically saturated, the set of formulas $\left\{(x)_{n}=t_{i_{n}}(b): n<\omega\right\}$ is realized in $\mathcal{M}$ by, say, $a \in M$. Then $a$ codes $M_{1}$.

Corollary 4.8: Suppose that $\mathcal{M}, \mathcal{N}$ are countable, arithmetically saturated models and that $\alpha: \operatorname{Aut}(\mathcal{M}) \longrightarrow \operatorname{Aut}(\mathcal{N})$ is an isomorphism. If $\mathcal{M}_{0} \prec \mathcal{M}$ and $\mathcal{M}_{0}$ is coded, then $\widetilde{\alpha}\left(\mathcal{M}_{0}\right)$ is coded in $\mathcal{N}$.

Proof. Suppose that $\mathcal{M}_{0} \prec \mathcal{M}$ is coded. By Lemma 4.7, let $\mathcal{M}_{1} \prec$ $\mathcal{M}$ be a superminimal end extension of $\mathcal{M}_{0}$. Then, by Lemma 4.3(2), $\widetilde{\alpha}\left(\mathcal{M}_{1}\right)$ is a superminimal end extension of $\widetilde{\alpha}\left(\mathcal{M}_{0}\right)$, so, by Lemma 4.7 , $\widetilde{\alpha}\left(\mathcal{M}_{0}\right)$ is coded in $\mathcal{N}$.

Lemma 4.9: Suppose that $\mathcal{M}, \mathcal{N}$ are countable, arithmetically saturated models and that $\alpha: \operatorname{Aut}(\mathcal{M}) \longrightarrow \operatorname{Aut}(\mathcal{N})$ is an isomorphism. If $\mathcal{M}_{1}, \mathcal{M}_{2} \preccurlyeq \mathcal{M}$ are coded and $\operatorname{SSy}\left(\mathcal{M}_{1}\right) \subseteq \operatorname{SSy}\left(\mathcal{M}_{2}\right)$, then $\operatorname{SSy}\left(\widetilde{\alpha}\left(\mathcal{M}_{1}\right)\right) \subseteq$ $\operatorname{SSy}\left(\widetilde{\alpha}\left(\mathcal{M}_{2}\right)\right)$.

Proof. This lemma was proved in [19, Coro. 5.3] under the additional hypotheses that $\mathcal{M}, \mathcal{N}$ are 2-Ramsey and that $\mathcal{M}_{1}, \mathcal{M}_{2}$ are finitely generated. The requirement that $\mathcal{M}, \mathcal{N}$ are 2-Ramsey was needed only because it also appeared in Proposition 4.2 of [19], which is our Lemma 4.4 except that Proposition 4.2 has the added hypothesis that $\mathcal{M}$ and $\mathcal{N}$ are 2-Ramsey.

Now suppose that $\mathcal{M}_{1}, \mathcal{M}_{2}$ are coded (as in the lemma) and that $\operatorname{SSy}\left(\mathcal{M}_{1}\right) \subseteq \operatorname{SSy}\left(\mathcal{M}_{2}\right)$. Then, by Lemma 4.7 , there are superminimal end extensions $\mathcal{M}_{3} \succ_{\text {end }} \mathcal{M}_{1}$ and $\mathcal{M}_{4} \succ_{\text {end }} \mathcal{M}_{2}$ and, therefore, $\operatorname{SSy}\left(\mathcal{M}_{3}\right)=\operatorname{SSy}\left(\mathcal{M}_{1}\right) \subseteq \operatorname{SSy}\left(\mathcal{M}_{2}\right)=\operatorname{SSy}\left(\mathcal{M}_{4}\right)$. Then, $\widetilde{\alpha}\left(\mathcal{M}_{3}\right)$, $\widetilde{\alpha}\left(\mathcal{M}_{4}\right)$ are finitely generated and, by Lemma $4.3(2)$, are end extensions of $\widetilde{\alpha}\left(\mathcal{M}_{1}\right), \widetilde{\alpha}\left(\mathcal{M}_{2}\right)$, repectively. Thus, we that $\operatorname{SSy}\left(\widetilde{\alpha}\left(\mathcal{M}_{1}\right)\right)=$ $\operatorname{SSy}\left(\widetilde{\alpha}\left(\mathcal{M}_{3}\right)\right) \subseteq \operatorname{SSy}\left(\widetilde{\alpha}\left(\mathcal{M}_{4}\right)\right)=\operatorname{SSy}\left(\widetilde{\alpha}\left(\mathcal{M}_{2}\right)\right)$.

The next lemma is a variation of Corollary 2.7 that takes place inside an arithmetically saturated model.

LEMMA 4.10: Suppose that $\mathcal{M}$ is an arithmetically saturated model, $\mathcal{M}_{0} \prec \mathcal{M}$ is coded and $X \in \mathrm{SSy}(\mathcal{M}) \backslash \operatorname{SSy}\left(\mathcal{M}_{0}\right)$. Then there is a coded $\mathcal{M}_{1} \succ_{\text {cf }} \mathcal{M}_{0}$ such that $X \notin \operatorname{SSy}\left(\mathcal{M}_{1}\right)$ and $\mathcal{M}_{1}$ has the $\omega$-property.

Proof. The main concern is that the appropriate variant of Lemma 2.6 can be proved. It is left to the reader to show that the proof of Lemma 2.6 can be appropriately modified. 
Remark: In this lemma, it is required that $\mathcal{M}_{1}$ have the $\omega$-property. In fact, it will have the stronger property that there are a coded $\mathcal{M}_{2} \succ_{\text {end }} \mathcal{M}_{1}$ and $a \in M_{2}$ such that $M_{1}=\sup ^{\mathcal{M}_{2}}\left\{(a)_{i}: i<\omega\right\}$.

The next lemma is a variant of Corollary 2.10 that takes place inside an arithmetically saturated model.

LEMma 4.11: Suppose that $\mathcal{M}$ is an arithmetically saturated model, $\mathcal{M}_{0} \prec \mathcal{M}$ is coded and $\mathfrak{X}=\operatorname{SSy}\left(\mathcal{M}_{0}\right)$. The following are equivalent:

(1) $\operatorname{Th}(\mathcal{M}) \notin \mathfrak{X}$;

(2) for every tall coded $\mathcal{M}_{1} \prec \mathcal{M}$ such that $\operatorname{SSy}\left(\mathcal{M}_{1}\right)=\mathfrak{X}$, there is a coded $\mathcal{M}_{2} \succcurlyeq_{\text {cf }} \mathcal{M}_{1}$ that is not generated by a set of indiscernibles.

Proof. Let $T=\operatorname{Th}(\mathcal{M})$.

$(1) \Longrightarrow(2)$ : Suppose that $T \notin \mathfrak{X}$. Let $\mathcal{M}_{1} \prec \mathcal{M}$ be tall and coded such that $\operatorname{SSy}\left(\mathcal{M}_{1}\right) \subseteq \mathfrak{X}$. By Lemma 4.10 , let $\mathcal{M}_{2} \prec \mathcal{M}$ be such that $\mathcal{M}_{1} \prec_{\text {cf }} \mathcal{M}_{2}, T \notin \operatorname{SSy}\left(\mathcal{M}_{2}\right)$ and $\mathcal{M}_{2}$ has the $\omega$-property. By Lemma $2.2, \mathcal{M}_{2}$ is uniformly $\omega$-logty. Since $T \notin \operatorname{SSy}\left(\mathcal{M}_{2}\right), \mathcal{M}_{2}$ is not recursively saturated, so, by Lemma $2.3, \mathcal{M}_{2}$ is not generated by a set of indiscernibles.

$(2) \Longrightarrow(1)$ : Suppose that $T \in \mathfrak{X}$. Let $\mathcal{M}_{1} \prec \mathcal{M}$ be coded, recursively saturated and be such that $\operatorname{SSy}\left(\mathcal{M}_{1}\right) \subseteq \operatorname{SSy}\left(\mathcal{M}_{0}\right)$. Then, $\mathcal{M}_{1}$ is tall, and every countable $\mathcal{M}_{2} \succcurlyeq_{\text {cf }} \mathcal{M}_{1}$ is recursively saturated and, hence, generated by a set of indiscernbles.

The next corollary says that those elementary submodels that code their own theories are recognizable.

Corollary 4.12: Suppose that $\mathcal{M}, \mathcal{N}$ are countable, arithmetically saturated models and that $\alpha: \operatorname{Aut}(\mathcal{M}) \longrightarrow \operatorname{Aut}(\mathcal{N})$ is an isomorphism. If $\mathcal{M}_{1} \preccurlyeq \mathcal{M}$ and $\operatorname{Th}(\mathcal{M}) \in \operatorname{SSy}\left(\mathcal{M}_{1}\right)$, then $\operatorname{Th}(\mathcal{N}) \in \operatorname{SSy}\left(\widetilde{\alpha}\left(\mathcal{M}_{1}\right)\right)$.

Proof. Suppose that $\mathcal{M}_{1} \preccurlyeq \mathcal{M}$ and $\operatorname{Th}(\mathcal{M}) \in \operatorname{SSy}\left(\mathcal{M}_{1}\right)$. Let $a \in M$ and $\mathcal{M}_{0}=\operatorname{Scl}(a)$ be such that $\operatorname{Th}(\mathcal{M}) \in \operatorname{SSy}\left(\mathcal{M}_{0}\right)$. Since $\widetilde{\alpha}\left(\mathcal{M}_{0}\right) \preccurlyeq$ $\widetilde{\alpha}\left(\mathcal{M}_{1}\right)$, it suffices to show that $\operatorname{Th}(\mathcal{N}) \in \operatorname{SSy}\left(\widetilde{\alpha}\left(\mathcal{M}_{0}\right)\right)$.

By $(2) \Longrightarrow(1)$ of Lemma 4.11 , there is a tall coded $\mathcal{M}_{2}$ such that $\operatorname{SSy}\left(\mathcal{M}_{2}\right)=\operatorname{SSy}\left(\mathcal{M}_{0}\right)$ and every coded $\mathcal{M}_{3} \preccurlyeq c f \mathcal{M}_{2}$ is generated by a set of indiscernibles.

For a contradiction, suppose that $\operatorname{Th}(\mathcal{N}) \notin \operatorname{SSy}\left(\mathcal{N}_{0}\right)$. Since $\mathcal{N}_{2}$ is tall and coded and $\operatorname{SSy}\left(\mathcal{N}_{2}\right)=\operatorname{SSy}\left(\mathcal{N}_{0}\right)$, then by By $(1) \Longrightarrow(2)$ of Lemma 4.11, there is a tall coded $\mathcal{N}_{3} \succcurlyeq \mathcal{N}_{2}$ that is generated by a set of indiscernibles. But then $\mathcal{M}_{3} \succcurlyeq \mathcal{M}_{2}$ is tall, coded and generated by a set of indiscernibles, which is a contradiction. 
Corollary 4.12 has a $*$-version, so it can be generalized from models $\mathcal{M}$ to models $(\mathcal{M}, a)$ as in part (a) of the next lemma. Let us say that a model $\mathcal{M}$ is a self-coder if $\operatorname{tp}(a) \in \operatorname{SSy}(\mathcal{M})$ whenever $a \in M$. Every recursively saturated model is a self-coder. Moreover, if $\mathcal{M}_{0}$ is countable, then, $\mathcal{M}_{0}$ is a self-coder iff there is a countable, recursively saturated $\mathcal{M}_{1} \succ \mathcal{M}_{0}$ such that $\operatorname{SSy}\left(\mathcal{M}_{1}\right)=\operatorname{SSy}\left(\mathcal{M}_{0}\right)$. Part (b) of the next lemma says that self-coders are recognizable.

Corollary 4.13: Suppose that $\mathcal{M}, \mathcal{N}$ are countable, arithmetically saturated models, $\alpha: \operatorname{Aut}(\mathcal{M}) \longrightarrow \operatorname{Aut}(\mathcal{N})$ is an isomorphism, and $\mathcal{M}_{1} \preccurlyeq \mathcal{M}$.

(a) If $a \in M_{1}, \widetilde{\alpha}(\operatorname{Scl}(a))=\operatorname{Scl}(b)$ and $\operatorname{tp}(a) \in \operatorname{SSy}\left(\mathcal{M}_{1}\right)$, then $\operatorname{tp}(b) \in \operatorname{SSy}\left(\widetilde{\alpha}\left(\mathcal{M}_{1}\right)\right)$.

(b) If $\mathcal{M}_{1}$ is a self-coder, then $\widetilde{\alpha}\left(\mathcal{M}_{1}\right)$ is a self-coder.

Lemma 4.14: Suppose that $\mathcal{M}_{0}$ is countable. Then $\mathcal{M}_{0}$ is recursively saturated iff each of the following holds:

(1) $\mathcal{M}_{0}$ is homogeneous;

(2) $\mathcal{M}_{0}$ is a self-coder;

(3) if $p(x) \in \operatorname{SSy}\left(\mathcal{M}_{0}\right)$ is a complete 1-type for $\operatorname{Th}\left(\mathcal{M}_{0}\right)$, then $p(x)$ is realized in $\mathcal{M}_{0}$.

Proof. $(\Longrightarrow)$ : By Proposition 1.3, every countable, recursively saturated model is homogeneous and, as previously mentioned, every recursively saturated model is a self-coder. Lastly, observe that (3) is a straightforward consequence of recursive saturation.

$(\Longleftarrow)$ : For the converse, suppose that $\mathcal{M}_{0}$ satisfies $(1)-(3)$. It follows from (2) that $\operatorname{Th}\left(\mathcal{M}_{0}\right) \in \operatorname{SSy}\left(\mathcal{M}_{0}\right)$. Thus, by Theorem 1.1, there is a countable, recursively saturated $\mathcal{M}_{1} \equiv \mathcal{M}_{0}$ such that $\operatorname{SSy}\left(\mathcal{M}_{1}\right)=$ $\operatorname{SSy}\left(\mathcal{M}_{0}\right)$. If $p(x)$ is a complete type, then (2) and (3) imply that $p(x)$ is realized in $\mathcal{M}_{0}$ iff $p(x) \in \operatorname{SSy}\left(\mathcal{M}_{0}\right)$. Thus, $\mathcal{M}_{0}$ and $\mathcal{M}_{1}$ realize exactly the same types. Since both are countable and homogeneous, they are isomorphic, so $\mathcal{M}_{0}$ is also recursively saturated.

The next lemma asserts that recursively saturated elementary submodels are recognizable.

Lemma 4.15: Suppose that $\mathcal{M}, \mathcal{N}$ are countable, arithmetically saturated models, $\alpha: \operatorname{Aut}(\mathcal{M}) \longrightarrow \operatorname{Aut}(\mathcal{N})$ is an isomorphism, and $\mathcal{M}_{0} \preccurlyeq \mathcal{M}$. If $\mathcal{M}_{0}$ is recursively saturated, then $\widetilde{\alpha}\left(\mathcal{M}_{0}\right)$ is recursively saturated. 
Proof. Suppose that $\mathcal{M}_{0}$ is recursively saturated, so that $\mathcal{M}_{0}$ satisfies (1) - (3) of Lemma 4.14. Let $\mathcal{N}_{0}=\widetilde{\alpha}\left(\mathcal{M}_{0}\right)$. Then, $\mathcal{N}_{0}$ is homogeneous by Lemma $4.5(1)$ and is a self-coder by Corollary 4.13(b). To get that $\mathcal{N}_{0}$ is recursively saturated, it remains to show that it has the property described in (3) of Lemma 4.14. So, consider a complete type $q(x) \in \operatorname{SSy}\left(\mathcal{N}_{0}\right)$, and let $b \in N$ realize $q(x)$ in $\mathcal{N}$. Let $a \in M$ be such that $\widetilde{\alpha}(\operatorname{Scl}(a))=\operatorname{Scl}(b)$. By Corollary 4.13(a), $\operatorname{tp}(a) \in \operatorname{SSy}\left(\mathcal{M}_{0}\right)$, and, therefore, by Lemma 4.14(3), $\mathcal{M}_{0}$ realizes $\operatorname{tp}(a)$. Let $a^{\prime} \in M_{0}$ be such that $\operatorname{tp}\left(a^{\prime}\right)=\operatorname{tp}(a)$. Then, $\operatorname{Scl}(a) \cong \operatorname{Scl}\left(a^{\prime}\right)$, so by Lemma 4.2 , $\operatorname{Scl}(b) \cong \widetilde{\alpha}\left(\operatorname{Scl}\left(a^{\prime}\right)\right)$. Let $b^{\prime} \in \widetilde{\alpha}\left(\operatorname{Scl}\left(a^{\prime}\right)\right)$ be such that $\operatorname{tp}\left(b^{\prime}\right)=\operatorname{tp}(b)$. But then $b^{\prime} \in N_{0}$ and $\operatorname{tp}\left(b^{\prime}\right)=q(x)$.

We can now complete the proof of Theorem 4. Suppose that $\mathcal{M}, \mathcal{N}$ are countable, arithmetically saturated models and that $\alpha: \operatorname{Aut}(\mathcal{M}) \longrightarrow$ $\operatorname{Aut}(\mathcal{N})$ is an isomorphism. Let $T=\operatorname{Th}(\mathcal{M})$ (so that $T^{\prime}$ is the Turingjump of $T)$. It suffice to prove that $T^{\prime} \leq_{T} \operatorname{Th}(\mathcal{N})^{\prime}$.

Since $T \in \operatorname{SSy}\left(\mathcal{M}_{0}\right)$ for every recursively saturated $\mathcal{M}_{0} \preccurlyeq \mathcal{M}$, we get from $(2) \Longrightarrow(1)$ of Lemma 3.1, that every recursively saturated $\mathcal{M}_{0} \preccurlyeq$ $\mathcal{M}$ has an elementary substructure $\mathcal{M}_{1} \prec \mathcal{M}_{0}$ such that $\operatorname{Lt}\left(\mathcal{M}_{1}\right) \cong$ $\mathcal{D}^{\prime}\left(T^{\prime}\right)$. Then Lemma 4.15 implies that

(*) every recursively saturated $\mathcal{N}_{0} \preccurlyeq \mathcal{N}$ has an elementary substructure $\mathcal{N}_{1} \prec \mathcal{N}_{0}$ such that $\operatorname{Lt}\left(\mathcal{N}_{1}\right) \cong \mathcal{D}^{\prime}\left(T^{\prime}\right)$.

Now suppose, for a contradiction, that $T^{\prime} \mathbb{Z}_{T} \operatorname{Th}(\mathcal{N})^{\prime}$. There is a countable Scott set $\mathfrak{X} \subseteq \operatorname{SSy}(\mathcal{N})$ such that $\operatorname{Th}(\mathcal{N}) \in \mathfrak{X}$ and $X^{\prime} \leq_{T}$ $\operatorname{Th}(\mathcal{N})^{\prime}$ for every $X \in \mathfrak{X}$. (See [23, Theorem VIII.2.17].) Hence, $T^{\prime} \not_{T}$ $X^{\prime}$ for every $X \in \mathfrak{X}$. By Theorem 1.1 , let $\mathcal{N}_{0}$ be recursively saturated model of $\operatorname{Th}(\mathcal{N})$ such that $\operatorname{SSy}\left(\mathcal{N}_{0}\right)=\mathfrak{X}$. Since $\mathfrak{X} \subseteq \operatorname{SSy}(\mathcal{N})$, we can assume that $\mathcal{N}_{0} \prec \mathcal{N}$. Then, by $(1) \Longrightarrow(2)$ of Theorem 3.1, there is no $\mathcal{N}_{1} \prec \mathcal{N}_{0}$ such that $\operatorname{Lt}\left(\mathcal{N}_{1}\right) \cong \mathcal{D}^{\prime}\left(T^{\prime}\right)$, contradicting $(*)$ and completing the proof of Theorem 4 .

We next prove Corollary 5 . We will actually prove something somewhat stonger since we will obtain completions $T_{0}, T_{1}, T_{2}, \ldots$ such that each $\mathcal{M}_{i}$ that we obtain is a model of $T_{i}$.

Let $\mathfrak{X}_{0}$ be a Scott set that is enumerated by some arithmetical $X$. Apply Theorem 1.2 to get, for each $n<\omega$, a completion $T_{n}$ of PA such that $T_{n} \equiv_{n} X^{(2 n)}$ and $\operatorname{Rep}\left(T_{n}\right)=\mathfrak{X}_{0}$. For any countable jump ideal $\mathfrak{X}$, Theorem 1.1 implies that there are models $\mathcal{M}_{n} \models T_{n}$ such that $\operatorname{SSy}\left(\mathcal{M}_{n}\right)=\mathfrak{X}$. Each $\mathcal{M}_{n}$ is arithmetically saturated since $\mathfrak{X}$ is a jump ideal. By Theorem 4, $\operatorname{Aut}\left(\mathcal{M}_{i}\right) \approx \operatorname{Aut}\left(\mathcal{M}_{j}\right)$ whenever $i<j<\omega$. 
§5. The Proof of Theorem 6. Lemma 4.15 showed that recursively saturated elementary submodels are recognizable. It is also the case that arithmetically saturated elementary submodels are recognizable.

Lemma 5.1: Suppose that $\mathcal{M}, \mathcal{N}$ are countable, arithmetically saturated models, $\alpha: \operatorname{Aut}(\mathcal{M}) \longrightarrow \operatorname{Aut}(\mathcal{N})$ is an isomorphism, and $\mathcal{M}_{0} \preccurlyeq \mathcal{M}$. If $\mathcal{M}_{0}$ is arithmetically saturated, then $\widetilde{\alpha}\left(\mathcal{M}_{0}\right)$ is arithmetically saturated.

Proof. There are several ways to prove this. One way is to use [8, Coro. 5.4] that asserts: If $\mathcal{M}_{0}$ is recursively saturated, then $\mathcal{M}_{0}$ is arithmetically saturated iff there is $g \in \operatorname{Aut}\left(\mathcal{M}_{0}\right)$ and an open $H<$ $\operatorname{Aut}\left(\mathcal{M}_{0}\right)$ such that for all $f \in \operatorname{Aut}\left(\mathcal{M}_{0}\right), f^{-1} g f \notin H$. We leave it to the reader to complete this proof.

Suppose that $T$ is any completion of PA. Theorem 1.1 implies that there is a (necessarily unique) recursively saturated model $\mathcal{M} \models T$ such that $\operatorname{SSy}(\mathcal{M})$ is the smallest jump ideal to which $T$ belongs. This model is arithmetically saturated and is elementarily embeddable in every arithmetically saturated model of $T$. We refer to this $\mathcal{M}$ as the minimal arithmetically saturated model of $T$. Minimal arithmetically saturated models are recognizable.

Corollary 5.2: Suppose that $\mathcal{M}, \mathcal{N}$ are countable, arithmetically saturated models, $\alpha: \operatorname{Aut}(\mathcal{M}) \longrightarrow \operatorname{Aut}(\mathcal{N})$ is an isomorphism, and $\mathcal{M}_{0} \preccurlyeq \mathcal{M}$. If $\mathcal{M}_{0}$ is a minimal arithmetically saturated model, then so is $\widetilde{\alpha}\left(\mathcal{M}_{0}\right)$.

Recall the following key result of [18, Theorem 3.8].

Lemma 5.3: Suppose that $\mathcal{M}, \mathcal{N}$ are countable, arithmetically saturated models, $\alpha: \operatorname{Aut}(\mathcal{M}) \longrightarrow \operatorname{Aut}(\mathcal{N})$ is an isomorphism, and $1 \leq$ $n<\omega$. If $(\mathbb{N}, \operatorname{Rep}(\operatorname{Th}(\mathcal{M}))) \models R T_{2}^{\mathrm{n}}$, then $(\mathbb{N}, \operatorname{Rep}(\operatorname{Th}(\mathcal{N}))) \models \mathrm{RT}_{2}^{\mathrm{n}}$.

The following is a rephrasing of Lemma 5.3 when $n=3$.

Corollary 5.4: Suppose that $\mathcal{M}, \mathcal{N}$ are countable, arithmetically saturated models, $\alpha: \operatorname{Aut}(\mathcal{M}) \longrightarrow \operatorname{Aut}(\mathcal{N})$ is an isomorphism. If $\mathcal{M}_{0} \prec \mathcal{M}$ and $\mathcal{N}_{0} \prec \mathcal{N}$ are the prime elementary submodels and $\operatorname{SSy}\left(\mathcal{M}_{0}\right)$ is a jump ideal, then $\operatorname{SSy}\left(\mathcal{N}_{0}\right)$ is a jump ideal. 
The $*$-version of the previous corollary implies that finitely generated models whose standard systems are jump ideals are recognizable. But this is also so for coded models.

LEMma 5.5: Suppose that $\mathcal{M}, \mathcal{N}$ are countable, arithmetically saturated models, $\alpha: \operatorname{Aut}(\mathcal{M}) \longrightarrow \operatorname{Aut}(\mathcal{N})$ is an isomorphism, and $\mathcal{M}_{0} \prec$ $\mathcal{M}$. If $\mathcal{M}_{0}$ is coded and $\operatorname{SSy}\left(\mathcal{M}_{0}\right)$ is a jump ideal, then $\operatorname{SSy}\left(\widetilde{\alpha}\left(\mathcal{M}_{0}\right)\right)$ is a jump ideal.

Proof. By Lemma 4.7, let $\mathcal{M}_{1} \prec \mathcal{M}$ be a superminimal end extension of $\mathcal{M}_{0}$. Then $\mathcal{M}_{1}$ is finitely generated and $\operatorname{SSy}\left(\mathcal{M}_{1}\right)=\operatorname{SSy}\left(\mathcal{M}_{0}\right)$. Also, $\widetilde{\alpha}\left(\mathcal{M}_{1}\right)$ is finitely generated, $\widetilde{\alpha}\left(\mathcal{M}_{1}\right) \succ_{\text {end }} \widetilde{\alpha}\left(\mathcal{M}_{0}\right)$ (by Lemma $4.3(2)$ ) and $\widetilde{\alpha}\left(\mathcal{M}_{1}\right)$ is finitely generated. Therefore, $\operatorname{SSy}\left(\widetilde{\alpha}\left(\mathcal{M}_{1}\right)\right)$ is a jump ideal and so is $\operatorname{SSy}\left(\widetilde{\alpha}\left(\mathcal{M}_{0}\right)\right)$.

Given an arithmetically saturated model $\mathcal{M}$, we let $j(\mathcal{M})$ be the cardinality of the set of all jump ideals $\mathfrak{X}$ for which there are $\mathcal{M}_{0} \preccurlyeq$ $\mathcal{M}_{1} \preccurlyeq \mathcal{M}$ such that $\mathcal{M}_{0}$ is coded, $\operatorname{SSy}\left(\mathcal{M}_{0}\right)=\mathfrak{X}$ and $\mathcal{M}_{1}$ is a minimal arithmetically saturated model.

Lemma 5.6: Suppose that $\mathcal{M}, \mathcal{N}$ are countable, arithmetically saturated models and $\operatorname{Aut}(\mathcal{M}) \cong \operatorname{Aut}(\mathcal{N})$. Then, $j(\mathcal{M})=j(\mathcal{N})$.

Proof. Immediate from Lemmas 4.9, 5.2 and 5.5.

We will say that a completion $T$ of PA is tight if there is $m<\omega$ such that whenever $\mathcal{M}$ is a countable, arithmetically saturated model of $T$, then $j(\mathcal{M})=m$. If $T$ is tight, then we let $j(T)$ be that $m$.

We now prove Theorem 6 . Fix $n<\omega$. Our goal is to obtain recursively equivalent completions $T_{0}, T_{1}, \ldots, T_{n}$ such that for each $i \leq n$, $T_{i}$ is tight and $j\left(T_{i}\right)=n+1-i$. Clearly, by Lemma 5.6, these theories will suffice to confirm Theorem 6 .

Consider any $A_{0} \subseteq \omega$ and then let $A_{0}, A_{1}, A_{2}, \ldots, A_{n} \subseteq \omega$ be such that $A_{0}<_{a} A_{1}<_{a} A_{2}<_{a} \cdots<_{a} A_{n}$ and whenever $A$ is such that $A_{0} \leq_{a} A \leq_{a} A_{n}$, then there is a unique $i \leq n$ such that $A \equiv_{a} A_{i}$. That there are $A_{1}, A_{2}, \ldots, A_{n}$ follows from a very special case of the theorem of Harding [3]. For each $i \leq n$, let $\mathcal{B}_{i}$ be the jump ideal $\left\{B \subseteq \omega: B \leq_{a} A_{i}\right\}$, and then let $X_{i} \in \mathcal{B}_{i}$ and $\mathfrak{X}_{i} \subseteq \mathcal{B}_{i}$ be such that $\mathfrak{X}_{i}$ is a Scott set, $A_{i} \in \mathfrak{X}_{i}$ and $X_{i}$ enumerates $\mathfrak{X}_{i}$. (See [23, Theorem VIII.2.17].) Let $X=X_{0} \oplus X_{1} \oplus \cdots \oplus X_{n} \oplus A_{n}$, so $\mathcal{B}_{n}$ is the smallest jump ideal containing $X$. By Theorem 1.2 , let $T_{i}$ be a completion of PA such that $T_{i} \equiv_{T} X$ and $\operatorname{Rep}\left(T_{i}\right)=\mathfrak{X}_{i}$. Each $T_{i} \in \mathcal{B}_{n}$, so by Theorem 1.1 , there is a countable, recursively saturated $\mathcal{M}_{i} \models T_{i}$ such 
that $\operatorname{SSy}\left(\mathcal{M}_{i}\right)=\mathcal{B}_{n}$. Clearly, $\mathcal{M}_{i}$ is a minimal arithmetically saturated model of $T_{i}$. It is clear that $j\left(\mathcal{M}_{i}\right) \leq n+1-i$ as that is the cardinality of the set $\left\{\mathcal{B}_{i}, \mathcal{B}_{i+1}, \ldots, \mathcal{B}_{n}\right\}$, which is the set of all jump ideals $\mathfrak{X}$ such that $\operatorname{Rep}\left(T_{i}\right) \subseteq \mathfrak{X} \subseteq \operatorname{SSy}\left(\mathcal{M}_{i}\right)$. Moreover, if $\mathcal{M}$ is any arithmetically saturated model of $T_{i}$, then $j(\mathcal{M}) \leq n+1-i$.

Thus, it remains to show that $j\left(T_{i}\right) \leq n+1-i$; that is, we must show that whenever $i \leq j \leq n$, then there is a coded elementary submodel of $\mathcal{M}_{i}$ whose standard system is $\mathcal{B}_{j}$. This is a consequence of the next lemma, which we state separately since it has its own interest.

LEMma 5.7: Suppose that $\mathcal{M}$ is arithmetically saturated, $\mathfrak{X}_{0}$ is a Scott set enumerated by some $X \in \operatorname{SSy}(\mathcal{M})$ and $\operatorname{Rep}(\operatorname{Th}(\mathcal{M})) \subseteq \mathfrak{X}_{0}$. Then there is a finitely generated $\mathcal{M}_{0} \prec \mathcal{M}$ such that $\operatorname{SSy}\left(\mathcal{M}_{0}\right)=\mathfrak{X}_{0}$.

Proof. For $n<\omega$, let $\mathcal{L}_{n}=\mathcal{L}_{\mathrm{PA}} \cup\left\{c_{0}, c_{1}, \ldots, c_{n-1}\right\}$, where the $c_{i}$ 's are new and distinct constant symbols. Thus, $\mathcal{L}_{0}=\mathcal{L}_{\mathrm{PA}}$. Let $\mathcal{L}=\mathcal{L}_{\mathrm{PA}} \cup\left\{c_{0}, c_{1}, c_{2}, \ldots\right\}$.

Suppose that $X \subseteq \omega$ enumerates $\mathfrak{X}_{0}$. (For the time being, no other assumptions are being made.) To simlify notation, let $X_{n}=(X)_{n}$.

We will construct a complete Henkin $\mathcal{L}$-theory $T \supseteq \operatorname{Th}(\mathcal{M})$, and then let $\mathcal{M}_{0}$ be the Henkin model of $T$. The theory $T$ will be the union of an increasing chain $T_{0} \subseteq T_{1} \subseteq T_{2} \subseteq \cdots$ of theories such that for each $n<\omega$,

(1) $T_{n}$ is an $\mathcal{L}_{n}$-theory and $T_{n} \subseteq \Pi_{n} \cup \Sigma_{n}$;

(2) for every $\mathcal{L}_{n}$-sentence $\sigma \in \Pi_{n}$, either $\sigma \in T_{n}$ or $\neg \sigma \in T_{n}$;

(3) $\left\{\left(b_{2 n}\right)_{i}=0: i \in X_{n}\right\} \cup\left\{\left(b_{2 n}\right)_{i}=1: i \in \omega \backslash X_{n}\right\} \subseteq T_{2 n+1}$;

(4) the sentence $\exists x \varphi_{n}(x) \rightarrow \varphi_{n}\left(b_{2 n+1}\right)$ is in $T_{2 n+2}$.

(5) $T_{n} \in \mathfrak{X}_{0}$;

(6) $T_{n} \cup \operatorname{Th}(\mathcal{M})$ is consistent.

Suppose that we have obtained the sequence $T_{0} \subseteq T_{1} \subseteq T_{2} \subseteq \cdots$ satisfying $(1)-(5)$ and that $T$ is its union.

It follows from (1) and (6) that $T$ is an $\mathcal{L}$-theory, from (2) that $T$ is complete, from (2) and (6) that $T \supseteq \operatorname{Th}(\mathcal{M})$, from (4) that $T$ is a Henkin theory, from (3) that $\operatorname{Rep}(T) \supseteq \mathfrak{X}_{0}$, and from (5) that $\operatorname{Rep}(T) \subseteq \mathfrak{X}_{0}$. Thus, we can let $\mathcal{M}_{1}$ be the $\mathcal{L}_{\mathrm{PA}}$-reduct of the Henkin model of $\operatorname{Th}(\mathcal{M})$. Hence, $\mathcal{M}_{1} \equiv \mathcal{M}$ and $\operatorname{SSy}\left(\mathcal{M}_{1}\right)=\mathfrak{X}_{0}$.

The construction of the sequence of theories proceeds by recursion.

Let $T_{0}=\Pi_{0} \cap \operatorname{Th}(\mathcal{M})$.

Suppose that $n=2 k+1$. Let $T_{n}^{\prime}=T_{n-1} \cup\left\{\left(b_{2 k}\right)_{i}=0: i \in X_{k}\right\} \cup$ $\left\{\left(b_{2 k}\right)_{i}=1: i \in \omega \backslash X_{k}\right\}$. Clearly, $T_{n}^{\prime} \in \mathfrak{X}_{0}$ and $T_{n}^{\prime} \cup \operatorname{Th}(\mathcal{M})$ is consistent. 
Let $T_{n}^{\prime \prime} \cup\left(\left(\Pi_{n} \cup \Sigma_{n}\right) \cap \operatorname{Th}(\mathcal{M})\right)$. Then, $T_{n}^{\prime \prime} \in \mathfrak{X}$ and $T_{n}^{\prime \prime}$ is consistent, so there is a complete $\mathcal{L}_{n}$-theory $T_{n}^{\prime \prime \prime} \supseteq T_{n}^{\prime \prime}$. Let $T_{n}=T_{n}^{\prime \prime \prime} \cap\left(\Pi_{n} \cup \Sigma_{n}\right)$.

Suppose that $n=2 k+2$. Let $T_{n}^{\prime}=T_{n-1} \cup\left\{\exists x \varphi_{n}(x) \rightarrow \varphi_{k}\left(b_{2 k+1}\right)\right\}$. Clearly, $T_{n}^{\prime} \in \mathfrak{X}$ and $T_{n}^{\prime} \cup \operatorname{Th}(\mathcal{M})$ is consistent. Now obtain $T_{n}$ from $T_{n}^{\prime}$ exactly as was done in the previous case.

We have shown how to obtain a model $\mathcal{M}_{1} \equiv \mathcal{M}$ such that $\operatorname{SSy}\left(\mathcal{M}_{1}\right)=$ $\mathfrak{X}_{0}$. (Thus, we have proved one of Scott's theorems.) We next indicate how the construction can be refined to a more effective one that yields a coded $\mathcal{M}_{1} \prec \mathcal{M}$.

First, we recall a theorem of Marker [17] that says that if a Scott set has an enumeration, then it has an effective enumeration. (More details about this, including a definition, can be found in [1, Chap. 19] or in [10].) It can be checked that if a Scott set has an enumeration in a jump ideal $\mathfrak{X}$, then it also has an effective enumeration in $\mathfrak{X}$. Thus, we can assume that $\mathfrak{X}_{0}$ is effectively enumerated by $X \in \operatorname{SSy}(\mathcal{M})$.

It can be checked that the construction of the sequence of theories is recursive in $X \oplus T$. Let $\Phi(x)$ be the set of 1-ary $\mathcal{L}_{\mathrm{PA}}$-formulas that are obtained by replacing each occurrence of $c_{n}$ by $(x)_{n}$. Then, $\Phi(x) \in \operatorname{SSy}(\mathcal{M})$ and is consistent with $\operatorname{Th}(\mathcal{M})$, so there is some $a \in M$ that satisfies $\Phi(x)$. Thus, without loss of generality, a codes $\mathcal{M}_{1}$. By Lemma 4.7 , let $\mathcal{M}_{0}$ be a superminimal end extension of $\mathcal{M}_{1}$ such that $\mathcal{M}_{0} \prec \mathcal{M}$.

We end this section by asking if Theorem 6 can be improved.

Question 5.8: Are there infinitely many, recursively equivalent completions $T_{0}, T_{1}, T_{2}, \ldots$ of PA such that whenever $i<j<\omega$ and $\mathcal{M}_{i}, \mathcal{M}_{j}$ are countable, arithmetically saturated models of $T_{i}, T_{j}$, respectively, then $\operatorname{Aut}\left(\mathcal{M}_{i}\right) \approx \operatorname{Aut}\left(\mathcal{M}_{j}\right)$ ?

In the absence of a positive answer to the previous question (or even following an unlikely negative answer), we could still ask the next question.

Question 5.9: Are there infinitely many countable, arithmetically saturated models no two of which have isomorphic automorphism groups but all of which have the same standard systems and recursively equivalent theories?

§6. Some Additional Results. A consequence of Theorems 1 and 4 is that there is a set $\mathcal{T}$ of $2^{\aleph_{0}}$ completions of PA such that if $\mathcal{M}$, $\mathcal{N}$ are nonisomorphic countable arithmetically saturated models and 
$\operatorname{Th}(\mathcal{M}), \operatorname{Th}(\mathcal{N}) \in \mathcal{T}$, then $\operatorname{Aut}(\mathcal{M}) \neq \operatorname{Aut}(\mathcal{N})$. The next corollary is a strengthening of this.

Corollary 6.1: Suppose that $\mathfrak{X}$ is a countable Scott set. There is a set $\mathcal{T}$ of $2^{\aleph_{0}}$ completions of PA such that every $T \in \mathcal{T}$ has $\operatorname{Rep}(T)=$ $\mathfrak{X}$ and if $\mathcal{M}, \mathcal{N}$ are nonisomorphic countable arithmetically saturated models such that $\operatorname{Th}(\mathcal{M}), \operatorname{Th}(\mathcal{N}) \in \mathcal{T}$, then $\operatorname{Aut}(\mathcal{M}) \neq \operatorname{Aut}(\mathcal{N})$.

Proof. Let $X \subseteq \omega$ enumerate $\mathfrak{X}$. For every $Y \geq_{T} X$, apply Theorem 1.1 to get a completion $T_{Y} \equiv_{T} Y$ such that $\operatorname{Rep}\left(T_{Y}\right)=\mathfrak{X}$. Then $\mathcal{T}=\left\{T_{Y}: Y \geq_{T} X\right\}$ has cardinality $2^{\aleph_{0}}$.

The proof of the main result of [19] showed that if $\mathcal{M}, \mathcal{N}$ are saturated models and $\operatorname{Aut}(\mathcal{M}) \cong \operatorname{Aut}(\mathcal{N})$, then $\operatorname{Th}(\mathcal{M})^{(\omega)} \equiv_{a} \operatorname{Th}(\mathcal{N})^{(\omega)}$. The conclusion can be improved in the manner of Theorem 4 with essentially the same proof as Theorem 4 .

TheOREM 6.2: If $\mathcal{M}, \mathcal{N}$ are saturated models and $\operatorname{Aut}(\mathcal{M}) \cong \operatorname{Aut}(\mathcal{N})$, then $\operatorname{Th}(\mathcal{M})^{\prime} \equiv_{T} \operatorname{Th}(\mathcal{N})^{\prime}$.

Theorem 6 also has a version for saturated models.

THEOREM 6.3: For each $n<\omega$, there are recursively equivalent completions $T_{0}, T_{1}, \ldots, T_{n}$ of PA such that whenever $i<j \leq n$ and $\mathcal{M}_{i} \models T_{i}, \mathcal{M}_{j} \models T_{j}$ are saturated models, then $\operatorname{Aut}\left(\mathcal{M}_{i}\right) \varsubsetneqq \operatorname{Aut}\left(\mathcal{M}_{j}\right)$.

Proof. Use the same theories as in the proof of Theorem 6.

There is another approach to proving the previous two theorems using the corresponding results for arithmetically saturated models and the following lemma, the proof of which we omit but which the reader should be able to work out.

Lemma 6.4: Suppose that $\mathcal{M}, \mathcal{N}$ are saturated models and that $\operatorname{Aut}(\mathcal{M}) \cong \operatorname{Aut}(\mathcal{N})$. If $\mathcal{M}_{0} \prec \mathcal{M}$ and $\mathcal{N}_{0} \prec \mathcal{N}$ are minimal arithmetically saturated models, then $\operatorname{Aut}\left(\mathcal{M}_{0}\right) \cong \operatorname{Aut}\left(\mathcal{N}_{0}\right)$.

\section{REFERENCES}

[1] C. J. Ash and J. Knight, Computable structures and the hyperarithmetical hierarchy, Studies in Logic and the Foundations of Mathematics, 144. NorthHolland Publishing Co., Amsterdam, 2000.

[2] Nicholas Bamber and Henryk Kotlarski, On interstices of countable arithmetically saturated models of Peano arithmetic, Math. Logic Quart. 43 (1997), $525-540$. 
[3] Christopher John Harding, Forcing in recursion theory, Ph.D. thesis, Swansea University, 1974

[4] Matt Kaufmann and James H. Schmerl, Saturation and simple extensions of models of Peano arithmetic, Ann. Pure Appl. Logic 27 (1984), 109-136.

[5] Matt Kaufmann and James H. Schmerl, Remarks on weak notions of saturation in models of Peano arithmetic, J. Symbolic Logic 52 (1987), 129-148.

[6] Richard Kaye, Models of Peano arithmetic, Oxford Logic Guides 15, Oxford Science Publications, Clarendon Press, Oxford, 1991.

[7] Richard Kaye, A Galois correspondence for countable recursively saturated models of Peano arithmetic, in [9, 293-312.

[8] Richard Kaye, Roman Kossak and Henryk Kotlarski, Automorphisms of recursively saturated models of arithmetic, Ann. Pure Appl. Logic 55 (1991), $67-99$.

[9] Richard Kaye and Dugald Macpherson, eds., Automorphisms of first-order structures, Oxford Science Publ., The Clarendon Press, Oxford University Press, New York, 1994.

[10] Julia F. Knight, Minimality and completions of PA, J. Symbolic Logic 66 (2001), 1447-1457.

[11] Roman Kossak, Models with the $\omega$-property, J. Symbolic Logic 54 (1989), 177-189.

[12] Roman Kossak, On extensions of models of strong fragments of arithmetic, Proc. Amer. Math. Soc. 108 (1990), 223-232.

[13] Roman Kossak and James H. Schmerl, Arithmetically saturated models of arithmetic (Special Issue: Models of Arithmetic), Notre Dame J. Formal Logic 36 (1995), 531-546.

[14] Roman Kossak and James H. Schmerl, The automorphism group of an arithmetically saturated model of Peano arithmetic, J. London Math. Soc. (2) 52 (1995), 235-244.

[15] Roman Kossak and James H. Schmerl, The Structure of Models of Peano Arithmetic, Oxford Logic Guides 50, Oxford Science Publications, Clarendon Press, Oxford, 2006.

[16] Daniel Lascar, The small index property and recursively saturated models of Peano arithmetic, in [9, 281-292.

[17] Angus Macintyre and David Marker, Degrees of recursively saturated models, Trans. Amer. Math. Soc. 282 (1984), 539-554.

[18] Ermek S. Nurkhaidarov, Automorphism groups of arithmetically saturated models, J. Symbolic Logic 71 (2006), 203-216.

[19] Ermek S. Nurkhaidarov and James H. Schmerl, Automorphism groups of saturated models of Peano Arithmetic, J. Symbolic Logic (to appear).

[20] James H. Schmerl, Automorphism groups of models of Peano arithmetic, J. Symbolic Logic 67 (2002), 1249-1264.

[21] James H. Schmerl, The automorphism group of a resplendent model, Arch. Math. Logic 51 (2012), 647-649.

[22] Dana Scott, Algebras of sets binumerable in complete extensions of arithmetic, in: Recursive function theory (J. C. E. Dekker, ed.), Amer. Math. Soc., Proc. Symp. Pure Math., vol. V, 1962, pp. 117-122.

[23] Stephen G. Simpson, Subsystems of second order arithmetic, (2nd ed.) Perspectives in Logic, Cambridge University Press, Cambridge, 2009. 
[24] George Wilmers, Some problems in set theory: non-standard models and their applications to model theory, Ph.D. thesis, Oxford University, 1975. 